\title{
Chapitre 6 \\ Comportement des enceintes de confinement
}

\subsection{Introduction}

Les caractéristiques des enceintes de confinement des REP français ont été présentées au paragraphe 2.3.2.3. Schématiquement, l'enceinte de confinement, quelquefois appelée troisième barrière de confinement en France, doit assurer le confinement des substances radioactives en cas de défaillance de la première (gaines des crayons combustibles) et de la deuxième barrière (circuit primaire de refroidissement). La meilleure étanchéité possible de l'enceinte de confinement doit donc être recherchée à la conception puis tout au long de la vie de l'installation.

L'enceinte de confinement est l'ultime barrière mise en place pour la protection des personnes du public à l'égard des substances radioactives libérées lors d'un accident à l'intérieur de l'enceinte. Elle comporte :

- le bâtiment du réacteur lui-même (décrit au § 2.3.2.3) ;

- les pénétrations dans ce bâtiment qui constituent des singularités de l'enceinte (tampon d'accès des matériels (TAM), sas du personnel, traversées des fluides, traversées « électriques », tube de transfert des assemblages combustibles) ;

- certaines tuyauteries constituant des extensions de l'enceinte, notamment les portions des circuits secondaires intérieures au bâtiment du réacteur (en 
particulier les tuyauteries principales d'alimentation en eau et les tuyauteries principales de vapeur, l'enveloppe du secondaire des générateurs de vapeur et les faisceaux de tubes des générateurs de vapeur [ces derniers faisant également partie de la deuxième barrière]).

Les pénétrations sont conçues pour conserver une étanchéité satisfaisante en situation accidentelle de dimensionnement. À cette fin, les traversées véhiculant des fluides sont équipées de clapets et de vannes d'isolement dont la fermeture est, pour la plupart d'entre elles, automatiquement commandée par le système de protection.

Par ailleurs, certains circuits constituent des extensions de la troisième barrière audelà de l'enceinte de confinement. Il s'agit des portions de circuits et des équipements associés qui répondent simultanément aux critères suivants :

- ils sont extérieurs au bâtiment du réacteur ;

- ils peuvent véhiculer hors de l'enceinte soit du fluide primaire, après un accident susceptible d'entraîner une détérioration des gaines des crayons combustibles (défaillance de la première barrière), soit l'atmosphère de l'enceinte après un accident susceptible de conduire simultanément à une dégradation de la première barrière et à un relâchement d'activité provenant du fluide primaire dans l'enceinte (défaillance de la deuxième barrière).

Par exemple, pour les réacteurs en exploitation (hors réacteur EPR), des circuits peuvent rester ouverts vers l'extérieur de l'enceinte ou être mis en service par ouverture des organes d'isolement de l'enceinte, de façon automatique lors d'un accident, pour la recirculation de l'eau des circuits d'injection de sécurité (RIS) et d'aspersion dans l'enceinte (EAS), ou en application des documents de conduite ou guides (notamment le guide d'intervention en accident grave).

Les circuits constituant des extensions de la troisième barrière de confinement peuvent conduire, en cas de défaillance (rupture de tuyauterie, etc.), à des transferts de substances radioactives dans les bâtiments périphériques du bâtiment du réacteur ou dans l'environnement (« bipasse » de la troisième barrière de confinement).

L'intégrité et l'étanchéité de la troisième barrière de confinement sont fondamentales pour éviter la dissémination de substances radioactives dans l'environnement dans les situations où la première et la deuxième barrières sont dégradées. C'est le cas notamment lors des accidents de perte de réfrigérant primaire (APRP) au cours desquels des substances radioactives sont relâchées dans le circuit primaire et dans l'enceinte de confinement. Leur dissémination dans le milieu extérieur dépend alors du taux de fuite de l'enceinte et des bipasses de celle-ci qui pourraient entraîner un relâchement direct de radioactivité dans l'environnement. La quantité de radioactivité qui s'échappe alors dépend de plusieurs phénomènes : la surpression dans l'enceinte provoquée par l'accident lui-même, le taux de fuite du bâtiment du réacteur à ce niveau de pression et la quantité de substances radioactives présentes dans l'enceinte sous forme de gaz ou d'aérosols. 
Pour les réacteurs en exploitation, en cas d'accident de fusion du cœur, l'augmentation de la pression à l'intérieur de l'enceinte exerce des contraintes importantes sur celle-ci (pouvant être supérieures à celles déterminées pour les conditions de fonctionnement retenues pour le dimensionnement de l'installation), conduisant à terme à la dégradation de ses parois. Les évolutions de la pression et de la température à l'intérieur de l'enceinte peuvent conduire à des contraintes telles que le taux maximum de fuite de l'enceinte retenu dans le cadre du dimensionnement peut être dépassé. Cette augmentation du taux de fuite peut résulter de détériorations des parois de l'enceinte (notamment, la perméabilité ou la fissuration des parois en béton peut augmenter sous l'effet des chargements thermiques et mécaniques appliqués au cours de la vie de l'installation puis lors de l'accident) ou bien d'une évolution des fuites au niveau des singularités de l'enceinte de confinement (traversées, sas, etc.).

Dans ce chapitre, le paragraphe 6.2 rappelle le comportement des enceintes de confinement dans les situations de dimensionnement pour les réacteurs en exploitation et le réacteur EPR, et traite en particulier des fuites au travers des parois de l'enceinte ou des différentes traversées de celle-ci pour les conditions de fonctionnement de dimensionnement.

Le paragraphe 6.3 s'intéresse au comportement des enceintes en situation d'accident de fusion du cœur pour les réacteurs en exploitation (réacteurs de 900 et de $1300 \mathrm{MWe})$.

Le paragraphe 6.4 présente pour les réacteurs en exploitation les situations de bipasse de la troisième barrière de confinement (ou de son extension) correspondant à la mise en communication directe du fluide primaire, ou de l'atmosphère de l'enceinte après un accident, avec l'extérieur de l'enceinte de confinement (bâtiments périphériques ou environnement), du fait d'une dégradation d'équipements (par exemple fuite ou rupture de tuyauterie ou d'équipement faisant partie des circuits constituant l'extension de la troisième barrière).

Les fuites via l'enveloppe de la partie secondaire des générateurs de vapeur et l'enveloppe externe des circuits d'eau ou de vapeur situées dans le bâtiment du réacteur ne sont pas abordées.

\subsection{Comportement des enceintes dans les situations de dimensionnement}

Le bâtiment du réacteur d'une installation du parc en exploitation est constitué :

- soit d'une enceinte avec une seule paroi en béton précontraint revêtue à l'intérieur d'une peau d'étanchéité métallique (tranches de $900 \mathrm{MWe}$ ) ;

- soit d'une enceinte avec deux parois en béton (tranches de 1300 et de $1450 \mathrm{MWe}$ ) : une paroi interne en béton précontraint sans peau d'étanchéité métallique et une paroi externe en béton armé. L'espace entre les deux parois est maintenu en dépression par un système de ventilation qui collecte et filtre les 
fuites venant de la paroi interne et des traversées avant leur rejet dans l'atmosphère (filtres absolus et pièges à iode).

Les caractéristiques de ces bâtiments sont précisées au paragraphe 2.3.2.3.

Il est à noter que l'enceinte de confinement du réacteur EPR comporte, comme pour les tranches de 1300 et de 1450 MWe, deux parois en béton avec un système de confinement dynamique ; de plus, l'intérieur de la paroi interne est revêtue d'une peau d'étanchéité métallique.

Pour l'étude des fuites des enceintes, il convient de distinguer :

- les fuites qui passent par des bâtiments périphériques ou par l'espace entre enceintes (tranches de $1300 \mathrm{MWe}$ et de $1450 \mathrm{MWe}$, EPR) et qui peuvent, soit faire l'objet de dépôts dans l'installation, soit être filtrées par les dispositifs de filtration équipant les systèmes de ventilation des bâtiments périphériques ou de l'espace entre enceintes ; ces fuites sont dénommées fuites « collectées » ;

- les fuites qui sont rejetées directement dans l'environnement (tranches de 900, de $1300 \mathrm{MWe}$ et de $1450 \mathrm{MWe}$ ); ces fuites sont dénommées fuites " non collectées ».

Pour le réacteur EPR, des dispositions de conception ont été prises visant à « pratiquement éliminer » les possibilités de fuites directes dans l'environnement.

\subsubsection{Enceintes à simple paroi (réacteurs de $900 \mathrm{MWe}$ )}

La figure 6.1 ci-dessous présente les différents chemins de fuites à travers l'enceinte :

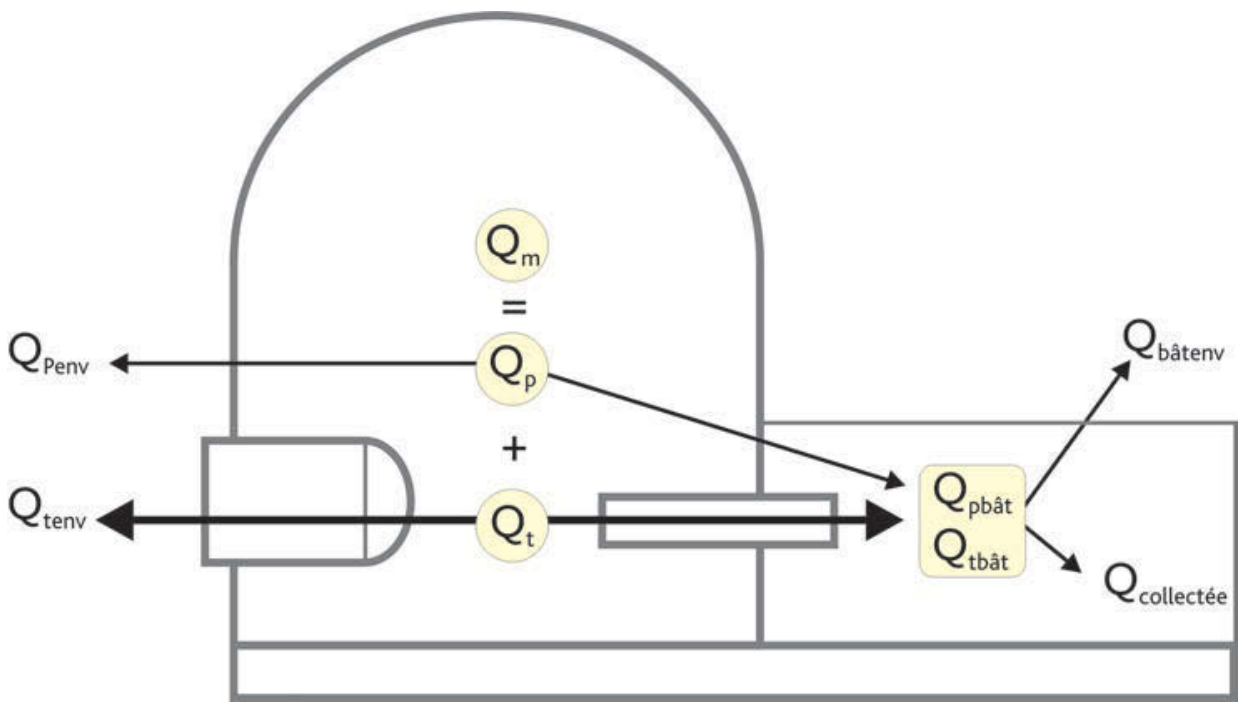

Figure 6.1. Les différents chemins de fuites à travers l'enceinte. 
Les fuites globales de l'enceinte $\left(Q_{m}\right)$ comportent les fuites par les traversées $\left(Q_{t}\right)$ et les fuites à travers la paroi de l'enceinte $\left(Q_{p}\right)$, recouverte sur la totalité de l'intrados de l'enceinte interne (radier compris) par la peau d'étanchéité métallique, ce qui se traduit par: $\mathrm{Q}_{\mathrm{m}}=\mathrm{Q}_{\mathrm{t}}+\mathrm{Q}_{\mathrm{p}}$.

Les fuites à travers la paroi de l'enceinte ou par les traversées se décomposent en deux familles principales:

- les fuites collectées (de débit $Q_{c}$ ) dans des bâtiments où existent des systèmes de filtration. Une partie des fuites passant dans ces bâtiments (notée $\mathrm{Q}_{\text {bâtenv }}$ ) peut cependant être relâchée dans l'atmosphère sans être filtrée, ce qui se traduit par $\mathrm{Q}_{\mathrm{c}}=\mathrm{Q}_{\text {pbât }}+\mathrm{Q}_{\text {tbât }}-\mathrm{Q}_{\text {bâtenv }}\left(\mathrm{Q}_{\text {tbât }}\right.$ et $\mathrm{Q}_{\text {pbât }}$ sont les fuites par les traversées et par la paroi collectées dans des bâtiments avec système de filtration);

- les fuites non collectées $\left(Q_{n c}\right)$ qui débouchent à l'extérieur de l'enceinte sans filtration $\left(Q_{n}=Q_{\text {penv }}+Q_{\text {tenv }}+Q_{\text {bâtenv }}\right)$, qui ont un impact prépondérant en termes de conséquences radiologiques $\left(\mathrm{Q}_{\text {tenv }}\right.$ et $\mathrm{Q}_{\text {penv }}$ sont les fuites directes par les traversées et par la paroi).

Les décrets d'autorisation de création (DAC) des REP 900 MWe mentionnent que l'enceinte de confinement doit être conçue pour supporter sans perte d'intégrité les sollicitations résultant d'un accident consistant en une rupture circonférentielle complète et soudaine d'une tuyauterie du circuit primaire avec séparation totale des extrémités (accident de perte de réfrigérant primaire étudié dans le cadre des accidents de dimensionnement). Dans les conditions de cet accident, le taux de fuite maximal de l'enceinte (par la paroi et les traversées) ne doit pas excéder 0,3\% par jour de la masse de gaz contenue dans l'enceinte.

Si la peau d'étanchéité métallique assure une bonne étanchéité, seules les singularités de l'enceinte que constituent les traversées sont susceptibles de conduire lors de l'accident à une augmentation significative des fuites de l'enceinte.

Les traversées de l'enceinte sont de plusieurs types:

- les traversées comportant une ou plusieurs tuyauteries traversant la paroi de l'enceinte ;

- les traversées utilisées pour le passage des conducteurs électriques ;

- les traversées utilisées pour les conduits de ventilation ;

- les traversées « réservées » pour permettre éventuellement le passage de tuyauteries ou de conducteurs non prévus lors de la conception initiale ;

- les traversées du radier de l'enceinte de confinement ;

- la traversée spécialement conçue pour le tube de transfert des assemblages combustibles ;

- la traversée spécialement conçue pour introduire ou sortir les gros matériels, appelée tampon d'accès des matériels (TAM) : une attention particulière a été 
portée par l'IRSN et par l'exploitant à l'étanchéité du tampon d'accès des matériels en conditions accidentelles (§ 6.3);

- la traversée conçue pour le sas utilisé par le personnel.

Les traversées de l'enceinte de confinement sont conçues pour assurer l'étanchéité de l'enceinte dans les conditions maximales de température et de pression à l'intérieur de l'enceinte lors des conditions de fonctionnement normal et lors des conditions de fonctionnement de référence (transitoires, incidents et accidents).

Les traversées des tuyauteries comprennent par exemple :

- un système doublé de barrières étanches constitué par la tuyauterie et le fourreau cylindrique en acier au carbone qui la contient ${ }^{1}$ et les vannes ou clapets antiretour d'isolement placés sur la tuyauterie à l'intérieur et à l'extérieur de l'enceinte ;

- un système de mesure périodique de leur taux de fuite ( $\mathrm{y}$ compris les vannes d'isolement) ;

- des dispositions permettant les essais périodiques du fonctionnement des vannes automatiques d'isolement.

Ces systèmes sont dimensionnés pour rester étanches dans toutes les conditions de fonctionnement citées plus haut, pour résister aux sollicitations liées à une rupture de tuyauterie et pour le séisme de dimensionnement. L'isolement de l'enceinte doit pouvoir être assuré dans tous les cas sans aucune intervention manuelle.

\subsubsection{Enceintes à double paroi (réacteurs de 1300 MWe et de 1450 MWe)}

Le confinement des réacteurs des paliers 1300 et 1450 MWe est réalisé par une enceinte à double paroi, avec :

- un confinement dynamique, grâce à un système de mise en dépression de l'espace entre enceintes (système EDE) qui récupère et filtre les fuites à travers le béton de la paroi interne ; après filtration, les fuites sont rejetées par la cheminée du bâtiment des auxiliaires nucléaires ;

- un confinement statique, reposant sur l'étanchéité de la paroi interne en béton précontraint et des traversées équipées de joints ou de vannes d'isolement des circuits.

La figure 6.2 et les définitions ci-après présentent la répartition des différentes catégories de fuites pour les enceintes à double paroi.

1. Le fourreau en acier carbone est soudé à l'intérieur de l'enceinte à la peau d'étanchéité métallique ; il est noyé et ancré dans le béton de la paroi. L'étanchéité de la traversée est assurée par :

- la soudure du fourreau sur la peau d'étanchéité ;

- le fourreau pour toute sa surface intérieure à l'enceinte de confinement ;

- la pièce de raccordement assurant la liaison entre le fourreau et la tuyauterie qu'il contient. 


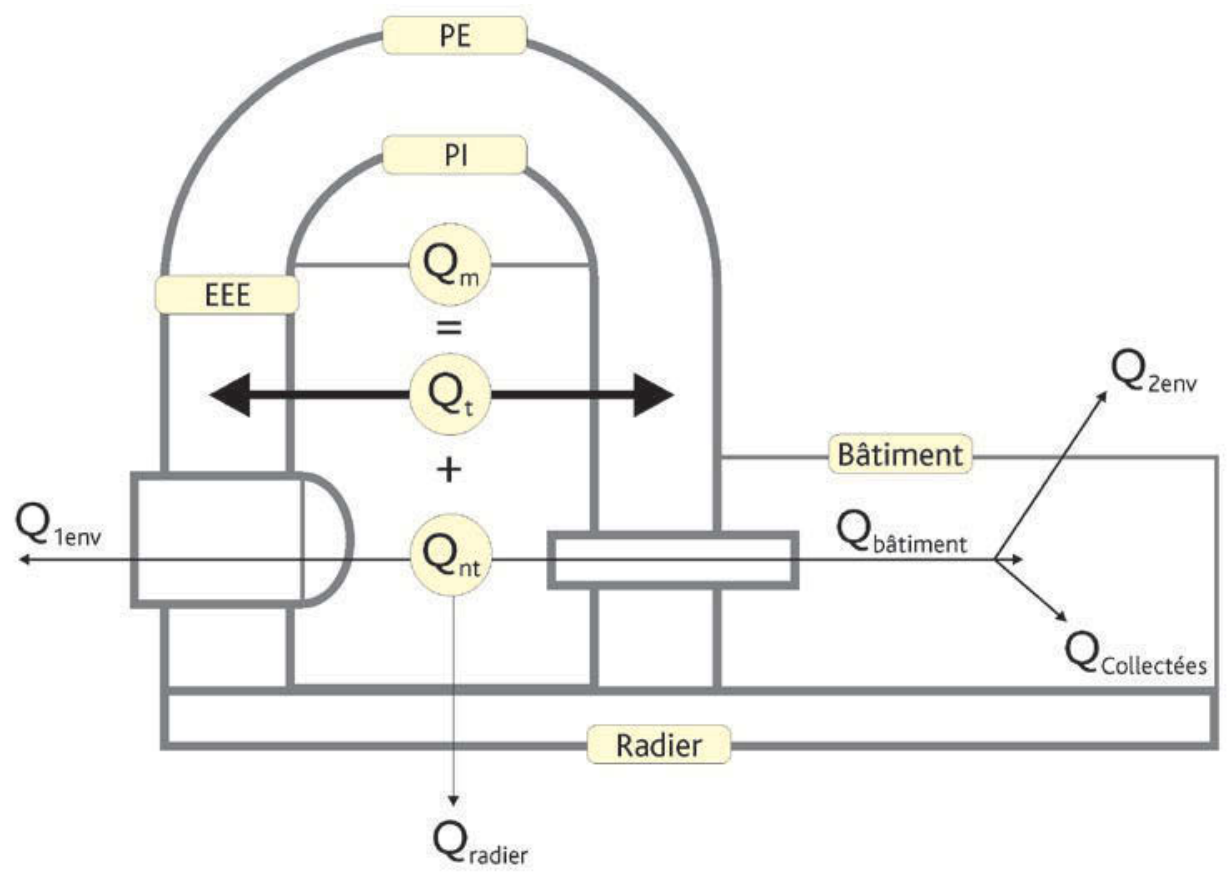

Figure 6.2. Répartition des différentes catégories de fuites pour les enceintes à double paroi.

Les fuites globales de l'enceinte $\left(Q_{m}\right)$ se répartissent entre les fuites récupérées $\left(Q_{t}\right)$ par le système EDE de mise en dépression de l'espace entre enceintes (EEE), et les fuites qui ne sont pas récupérées par ce système $\left(Q_{n t}\right)$, ce qui se traduit par: $Q_{m}=Q_{t}+Q_{n t}$.

Les fuites non récupérées par le système $\operatorname{EDE}\left(\mathrm{Q}_{\mathrm{nt}}\right)$ sont composées :

- des fuites qui passent par des traversées débouchant directement à l'extérieur (le tampon d'accès des matériels par exemple) : $Q_{1 \text { env }}$;

- des fuites par le radier : $Q_{\text {radier }}$;

- des fuites arrivant dans des bâtiments équipés d'une filtration, $Q_{\text {batiment }}$, dont une partie peut être relâchée dans l'atmosphère $\left(\mathrm{Q}_{2 \text { env }}\right)$.

Ainsi, les fuites qui ne sont pas récupérées par le système EDE peuvent s'exprimer de la façon suivante : $\mathrm{Q}_{\mathrm{nt}}=\mathrm{Q}_{\text {1env }}+\mathrm{Q}_{\text {radier }}+\mathrm{Q}_{\text {bâtiment }}$ avec $\mathrm{Q}_{\text {bâtiment }}=\mathrm{Q}_{\text {zenv }}+\mathrm{Q}_{\text {collectée }}$.

Les fuites non collectées $\left(Q_{n c}\right)$ sont celles qui débouchent dans l'environnement $\left(\mathrm{Q}_{\mathrm{nc}}=\mathrm{Q}_{\mathrm{nt}}-\mathrm{Q}_{\text {radier }}-\mathrm{Q}_{\text {collectée }}=\mathrm{Q}_{\text {1env }}+\mathrm{Q}_{\text {2env }}\right)$; elles ont un impact prépondérant en termes de conséquences radiologiques. Les traversées de l'enceinte ne débouchant pas dans l'EEE sont donc des éléments sensibles.

Pour les tranches de 1300 MWe et de 1450 MWe, les décrets d'autorisation de création (DAC) mentionnent que :

- le taux de fuite de l'enceinte interne $\left(\mathrm{Q}_{\mathrm{m}}\right)$ ne doit pas excéder 1,5\% par jour de la masse de gaz contenue dans l'enceinte interne dans les conditions de pression 
et de température d'un accident consistant en une rupture complète et soudaine d'une tuyauterie du circuit primaire avec séparation totale des extrémités (accident de perte de réfrigérant primaire étudié dans la cadre des accidents de dimensionnement) ;

- le taux de fuite de l'enceinte externe ne doit pas excéder $1 \%$ par jour de la masse totale de gaz contenue dans le volume limité par le parement interne de l'enceinte externe pour une dépression dans l'espace entre enceintes de 3 mbar.

Le débit de ventilation du système EDE est fixé pour assurer dans les conditions de l'accident de perte de réfrigérant primaire mentionné plus haut une dépression d'environ 15 mbar dans l'espace entre enceintes. Ce débit permet de conserver dans ces conditions une dépression dans l'espace entre enceintes et d'éviter toute fuite de l'espace entre enceintes vers l'extérieur autrement que par le système EDE pour le débit de fuite maximal de l'enceinte interne spécifié dans les DAC (voir ci-dessus) et pour une vitesse du vent d'environ $120 \mathrm{~km} / \mathrm{h}$ (la dépression permet dans ces conditions de contrecarrer l'effet de succion dû au vent).

L'évolution des fuites globales de l'enceinte et de leur répartition dépend, en situation accidentelle, de l'évolution des fuites de l'enceinte interne dues à l'augmentation de la perméabilité et de la fissuration du béton, des fuites par les traversées de l'enceinte, mais aussi des fuites de l'enceinte externe fonction de l'efficacité de collecte du système EDE.

\subsubsection{Enceintes à double paroi des réacteurs EPR}

Comme indiqué plus haut, pour les réacteurs EPR, des dispositions de conception ont été prises visant à « pratiquement éliminer » les possibilités de fuites directes dans l'environnement.

Ainsi, il ne doit pas y avoir de chemin de fuite directe de l'enceinte de confinement vers l'extérieur. Les tuyauteries susceptibles de transporter des substances radioactives à l'extérieur de l'enceinte de confinement doivent conduire à des bâtiments périphériques présentant des capacités de confinement adéquates. Certains des bâtiments périphériques au bâtiment du réacteur, notamment le bâtiment des auxiliaires nucléaires, les bâtiments des auxiliaires de sauvegarde et le bâtiment du combustible, participent à la fonction de confinement (maintien dans ces bâtiments d'un confinement dynamique à l'aide de dispositifs de ventilation et de filtration pour les aérosols et pour l'iode).

Le dimensionnement du confinement prend en compte les accidents de fusion du cœur avec défaillance de la cuve du réacteur à basse pression. En cas d'accident de ce type, le confinement doit être assuré pendant 12 heures même en l'absence de refroidissement de l'enceinte de confinement.

Les critères suivants ont été retenus :

- le taux de fuite de l'enceinte interne ne doit pas excéder 0,3\% par jour de la masse de gaz contenue dans l'enceinte interne dans les conditions des accidents considérés dans le dimensionnement (accidents de perte de réfrigérant primaire et accidents de fusion du cœur) ; 
- le taux de fuite de l'enceinte externe ne doit pas excéder 1,5\% par jour de la masse totale de gaz contenue dans le volume limité par le parement interne de l'enceinte externe pour une dépression dans l'espace entre enceintes de 6,2 mbar (la dépression de 6,2 mbar permet de compenser les effets de succion liés à un vent de $80 \mathrm{~km} / \mathrm{h})$.

Dans le cas des accidents considérés dans le dimensionnement, y compris les accidents de fusion du cœur, le système EDE doit assurer une dépression de l'espace entre enceintes, collecter et filtrer les fuites de l'enceinte interne qui est en surpression du fait de l'accident.

\subsubsection{Contrôle de l'intégrité et de l'étanchéité des enceintes de confinement}

La fonction de confinement des substances radioactives est assurée, au-delà des deux premières «barrières », par l'intégrité et l'étanchéité de l'enceinte de confinement et de ses traversées dans les différentes conditions de pression et de température envisagées.

L'enceinte fait l'objet :

- d'essais de résistance mécanique par mise en pression ;

- d'essais d'étanchéité de deux types:

- des essais globaux par mise en pression de l'enceinte ;

- des essais partiels par mise en pression locale des traversées et des systèmes d'isolement des fuites.

\subsubsection{Essais de résistance mécanique}

\section{- Épreuve initiale}

Avant la mise en service d'un réacteur, le bâtiment du réacteur est soumis à une épreuve de résistance, effectuée par mise en pression progressive de l'enceinte à la température ambiante jusqu'à la pression d'épreuve. Les mesures fournies par les dispositifs d'auscultation mécanique sont enregistrées lors de la pressurisation et de la dépressurisation de l'enceinte.

Pour les réacteurs de 900 MWe à peau métallique d'étanchéité, la pression d'épreuve est prise égale à 1,15 fois la pression relative de calcul de l'enceinte, pour tenir compte de la poussée thermique de la peau métallique d'étanchéité en cas d'accident de perte de réfrigérant primaire.

Pour l'enceinte interne du réacteur EPR, également munie d'une peau métallique, cette poussée conduit à une pression d'épreuve initiale égale à 1,10 fois la pression de calcul.

Pour les réacteurs sans peau métallique d'étanchéité (réacteurs de $1300 \mathrm{MWe}$ et 1450 MWe des paliers P4, P'4 et N4), la pression d'épreuve est la pression de calcul. 


\section{Essais périodiques}

Après l'épreuve initiale, l'enceinte fait l'objet d'essais mécaniques, à la pression de calcul, lors de la visite complète initiale $(\mathrm{VCl})$ et lors de chacune des visites décennales. Les mesures fournies par les dispositifs d'auscultation mécanique sont enregistrées lors de la pressurisation et de la dépressurisation de l'enceinte.

Des mesures d'auscultation effectuées régulièrement en service permettent de suivre l'évolution des déformations différées de l'ouvrage hors pression (retrait et fluage du béton).

\subsubsection{Essais d'étanchéité}

\section{- Essais globaux d'étanchéité de l'enceinte (essais dits de type A)}

Les essais globaux d'étanchéité ont pour objet la mesure du taux de fuite global pour les enceintes de confinement avec revêtement métallique (réacteurs de $900 \mathrm{MWe}$ et EPR) et du taux de fuite de la paroi interne de l'enceinte et de ses composants pour les réacteurs à double paroi sans revêtement métallique (réacteurs de $1300 \mathrm{MWe}$ et de $1450 \mathrm{MWe}$ ). Ils sont réalisés à la fin de la construction (essais préopérationnels), avant le premier chargement du cœur, puis tous les dix ans.

Les essais globaux d'étanchéité sont effectués à la pression de calcul de l'enceinte.

La détermination du taux de fuite est effectuée en mesurant la pression de l'air sec (corrigée de la pression partielle de vapeur d'eau obtenue par mesure de l'hygrométrie) contenu dans l'enceinte, en mesurant la température en différents endroits intérieurs à l'enceinte et en appliquant la loi des gaz parfaits : la variation de la quantité PV/T au cours du temps permet de connaître la perte de masse au cours du temps et donc le taux de fuite défini comme le rapport de la masse d'air s'étant échappée de l'enceinte pendant une durée de 24 heures sur la masse totale d'air sous pression présente dans l'enceinte.

Le taux de fuite (incertitudes comprises) est comparé au critère, égal à $0,162 \% / \mathrm{j}$ pour les enceintes à peau métallique (réacteurs de 900 MWe et EPR) et égal à 1,125 \%/j pour les enceintes internes des réacteurs à enceinte à double paroi sans peau métallique (réacteurs de $1300 \mathrm{MWe}$ et de $1450 \mathrm{MWe}$ ).

\section{- Essais globaux d'étanchéité des enceintes externes des réacteurs de $1300 \mathrm{MWe}$, de 1450 MWe et du réacteur EPR}

Un essai global d'étanchéité de l'enceinte externe et de ses traversées est réalisé au cours des essais du circuit de mise en dépression de l'espace entre enceintes, préalablement aux essais globaux de type A. 
Schématiquement, cet essai est réalisé en mettant en dépression (par rapport à la pression atmosphérique) l'espace entre parois de l'enceinte par le système EDE et en mesurant le débit rejeté par ce circuit, qui est égal au débit entrant dans l'espace entre les parois de l'enceinte.

Pour les réacteurs de $1300 \mathrm{MWe}$ et de $1450 \mathrm{MWe}$, le critère d'acceptabilité de l'essai est que le débit mesuré soit inférieur à $1 \%$ par jour de la masse d'air contenue dans l'espace délimité par l'intrados de l'enceinte externe pour une dépression dans l'espace entre enceintes de 3 mbar.

\section{- Essais partiels d'étanchéité des pénétrations dans le bâtiment du réacteur}

Des essais partiels d'étanchéité sont réalisés pour les pénétrations dans le bâtiment du réacteur (dans la paroi de l'enceinte pour les réacteurs de $900 \mathrm{MWe}$ et dans la paroi interne pour les réacteurs de $1300 \mathrm{MWe}$ et de $1450 \mathrm{MWe}$ et pour le réacteur EPR) afin de détecter et de mesurer les fuites locales pouvant affecter certaines pénétrations et les organes d'isolement associés. Ces essais sont subdivisés en essais dits de type $B$ et en essais dits de type $C$.

Les essais de type $B$ concernent :

- les traversées « électriques » (y compris celles des sas) ;

- les dispositifs d'étanchéité des portes des sas utilisés par le personnel, y compris les pénétrations pour la commande des portes ;

- le dispositif d'étanchéité du tampon de l'accès des matériels ;

- les traversées munies de fonds pleins démontables avec joints (en particulier la traversée utilisée pour le transfert du combustible).

Les essais de type $C$ concernent les organes d'isolement des tuyauteries traversant l'enceinte, autres que ceux qui font l'objet des essais de type B et autres que ceux des circuits secondaires qui sont considérés comme des circuits fermés dans l'enceinte et maintenus en surpression par rapport à l'atmosphère de celle-ci.

Essais d'étanchéité des tronçons de circuits extérieurs à l'enceinte et qui sont susceptibles de véhiculer des fluides contaminés hors de l'enceinte lors d'accidents avec défaillance des deux premières barrières (extensions de la troisième barrière, voir $\S 6.1$ ).

Pour les réacteurs en exploitation, il s'agit des circuits d'injection de sécurité (RIS), d'aspersion dans l'enceinte (EAS) ainsi que du système de contrôle de la teneur en hydrogène en cas d'accident (ETY).

Les essais d'étanchéité des tronçons extérieurs à l'enceinte des circuits RIS, EAS et ETY sont réalisés avec une périodicité égale à celle des essais globaux d'étanchéité. 


\subsection{Comportement mécanique des enceintes en cas d'accident de fusion du cœur}

\subsubsection{Introduction}

Lors d'un accident grave, l'enceinte de confinement du réacteur concerné pourrait être soumise à plusieurs types de sollicitations dépassant les conditions de pression et de température retenues pour son dimensionnement. Ces sollicitations seraient induites par les phénomènes physiques décrits dans les paragraphes 5.1 à 5.3, qui interviennent lors des différentes phases de la dégradation du cœur, notamment une combustion éventuelle d'hydrogène produit lors de la dégradation des gaines des crayons combustibles ou lors du renoyage du cœur dégradé, une explosion de vapeur dans la cuve ou en dehors de celle-ci et l'augmentation lente de la pression dans l'enceinte pendant l'interaction du corium avec le béton du radier.

Des travaux approfondis ont été entrepris ou sont en cours pour apprécier le comportement des enceintes de confinement des réacteurs français sous l'effet de telles sollicitations hors du domaine de dimensionnement des enceintes. L'approche est différente suivant le type d'enceinte de confinement, mais l'objectif est le même car il s'agit in fine de déterminer le débit de fuite de l'enceinte vers l'environnement au-delà des conditions de pression et de température retenues pour son dimensionnement.

Le problème est complexe et il faut faire appel pour le résoudre à des études mécaniques sophistiquées comportant notamment des évaluations théoriques des contraintes et des déformations de l'enceinte, complétées d'études expérimentales permettant de confirmer les évaluations théoriques. Des efforts de recherche et développement sont également nécessaires, notamment dans le domaine de la fissuration du béton soumis aux chargements correspondant à un accident de fusion du cœur. À ce sujet, EDF a engagé d'importants travaux expérimentaux (voir la maquette MAEVA décrite au § 6.3.3.2) et théoriques [1] applicables principalement aux enceintes à double parois des réacteurs de $1300 \mathrm{MWe}$.

Des études ont également été effectuées par l'IRSN en support à la réalisation de ses études probabilistes de sûreté (EPS) de niveau 2 pour les réacteurs de $900 \mathrm{MWe}$ et de 1300 MWe .

À titre d'illustration, un aperçu relativement détaillé de ces études et des résultats obtenus est présenté dans le paragraphe 6.3.2 pour le cas des enceintes des réacteurs de $900 \mathrm{MWe}$. Des études sont également développées à l'IRSN pour les enceintes des réacteurs de $1300 \mathrm{MWe}$. La démarche utilisée pour les calculs relatifs à ce type d'enceinte est présentée au paragraphe 6.3.3.

\subsubsection{Comportement mécanique des enceintes des centrales REP de $900 \mathrm{MWe}$}

Les études entreprises reposent sur des calculs non linéaires par la méthode des éléments finis. La construction de modèles représentant l'enceinte à des échelles différentes (démarche « multi-échelles ») permet d'appréhender le comportement de l'enceinte 
à différents niveaux de détail, en distinguant la zone courante, la zone du tampon d'accès des matériels (TAM), enfin la fermeture de ce dernier. Cette démarche permet de reproduire de façon réaliste les différents phénomènes thermomécaniques en conservant des temps et des coûts de calcul raisonnables.

Les calculs mécaniques ont été réalisés successivement en utilisant des maillages adaptés à la finesse souhaitée de la modélisation : modèle global complet de l'enceinte de confinement, modèle quart d'enceinte, modèle local de la traversée du TAM et modèle restreint du fourreau du TAM $[3,4]$. Mais, au préalable, il a fallu définir des profils enveloppes des chargements résultant des sollicitations subies par l'enceinte de confinement lors d'un accident de fusion du cœur.

\subsubsection{Sélection des scénarios d'accident de fusion du cœur retenus dans les études (évolutions de pression et de température dans l'enceinte)}

Les études mécaniques réalisées par l'IRSN dans le cadre de son EPS de niveau 2 relative aux centrales REP de 900 MWe ont visé à évaluer la réponse de l'enceinte de confinement à des sollicitations quasi statiques, correspondant à un pic de pression ou à une montée lente en pression [1].

Des calculs linéaires réalisés pour plusieurs scénarios d'accident de fusion du cœur ont permis de déterminer le scénario qui conduit aux dégradations les plus importantes de l'enceinte de confinement. Il s'agit du scénario dénommé AF qui comporte trois phases (figure 6.3) :

- une phase de préchargement thermique correspondant à la dégradation du cœur ; les instants P1 et P2 indiqués sur la figure 6.3 correspondent respectivement au début et à la fin de cette phase ;

- la phase du pic de pression et de température correspondant à la combustion adiabatique isochore de l'hydrogène produit par l'oxydation du cœur ; l'instant P3 indiqué sur la figure 6.3 correspond au pic;

- une phase de montée lente de la pression et de la température correspondant à l'interaction corium-béton avec mise en contact du corium avec l'eau du puisard ; une grande quantité de gaz chauds est en effet produite lors de cette interaction, ce qui provoque une montée de la pression et de la température dans l'enceinte ; les instants P4 et P5 indiqués sur la figure 6.3 correspondent respectivement au début et à la fin de cette phase; P5 a été fixé à une pression supposée au-delà de la pression ultime de tenue de l'enceinte.

Une étude paramétrique préalable réalisée pour différentes valeurs du pic de pression et de température à l'instant P3 a permis de déterminer une valeur de pic de pression voisine de 11,4 bars absolus (2,3 fois la pression de dimensionnement de l'enceinte). Cette pression correspond à celle produite par la combustion adiabatique isochore de $125 \%$ de la quantité d'hydrogène maximale générée par l'oxydation du cœur. Le choix des valeurs de pression et de température extrêmes du scénario AF permet d'obtenir un majorant des chargements mécaniques de l'enceinte pour les scénarios d'accident de fusion du cœur traités dans l'EPS de niveau 2 de l'IRSN. Dans le but de quantifier les 


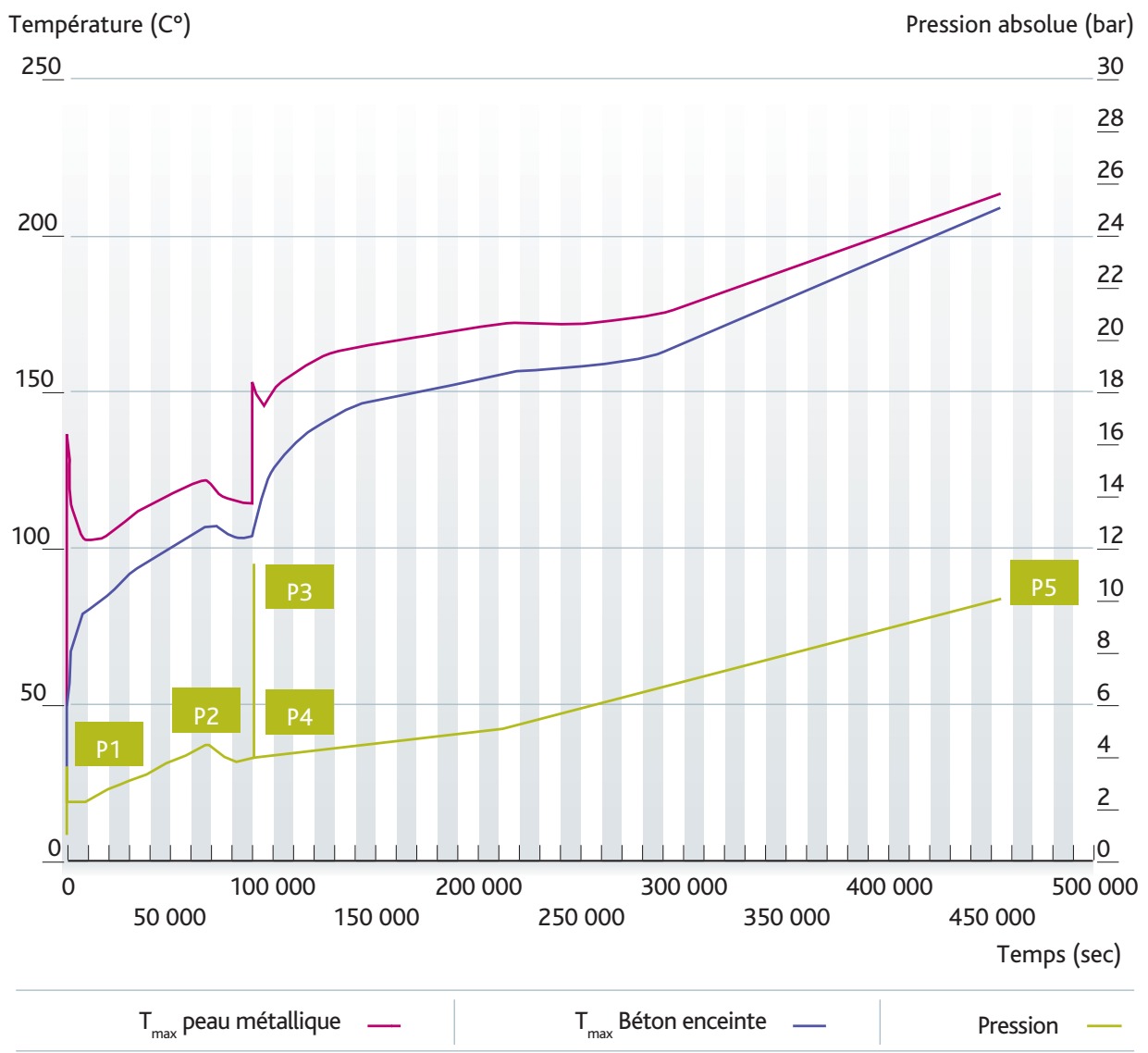

Figure 6.3. Évolution de la pression et de la température de l'enceinte pour le scénario AF.

effets thermiques de l'accident sur le comportement mécanique de l'enceinte, le scénario AF comprend par ailleurs deux cinétiques de montée en température différentes (figure 6.3), l'une à l'instant P3 correspondant à une montée rapide de la température de l'enceinte, l'autre entre les instants P4 et P5 (phase d'interaction corium-béton) correspondant à une montée lente de cette température, afin de vérifier la possibilité d'extrapoler les résultats de l'étude effectuée aux autres scénarios d'accidents de fusion du cœur ayant des profils temporels de température dans l'enceinte différents.

\subsubsection{Modèle global complet de l'enceinte de confinement (calcul de l'état initial de l'enceinte)}

Le calcul du comportement de l'enceinte de confinement lors d'un scénario d'accident de fusion du cœur de type AF nécessite de connaître de la façon la plus réaliste possible l'état de l'ouvrage avant l'accident sous les effets des phénomènes de retrait-fluage. L'âge de l'enceinte a été pris égal à 30 ans. Le calcul de la précontrainte et du fluage pour déterminer l'état de la structure à 30 ans a été réalisé sur une enceinte représentative d'une centrale REP de $900 \mathrm{MWe}$. Ce calcul, dont la modélisation géométrique et les 
chargements mécaniques sont présentés ci-après, a servi de base à l'ensemble des calculs réalisés avec les différentes modélisations décrites aux paragraphes 6.3.2.3 à 6.3.2.5.

La position et la mise en tension des câbles de précontrainte ne présentent pas des symétries suffisantes pour s'affranchir d'un calcul sur une enceinte complète $\left(360^{\circ}\right)$.

Le maillage utilisé reproduit les différents constituants de l'enceinte, le béton de la paroi, le ferraillage passif, la peau métallique, le béton de protection posé sur le radier et, avec une modélisation simplifiée, la virole de l'accès des matériels avec le fourreau, les brides et le fond. Tous les câbles de précontrainte, leur géométrie et leurs déviations, notamment autour de l'accès des matériels et des deux sas « personnel ", sont modélisés d'une façon précise. Le modèle reproduit aussi les effets du sol et du remblai. Les figures 6.4 (première figure de gauche) et 6.5 présentent les maillages utilisés.

L'enceinte en béton est soumise à son poids propre et à la précontrainte des câbles, calculée en tenant compte des différentes phases de mise en tension, des différentes causes de perte de tension (frottement, recul des têtes d'ancrage, etc.) et des particularités géométriques d'ensemble des câbles.

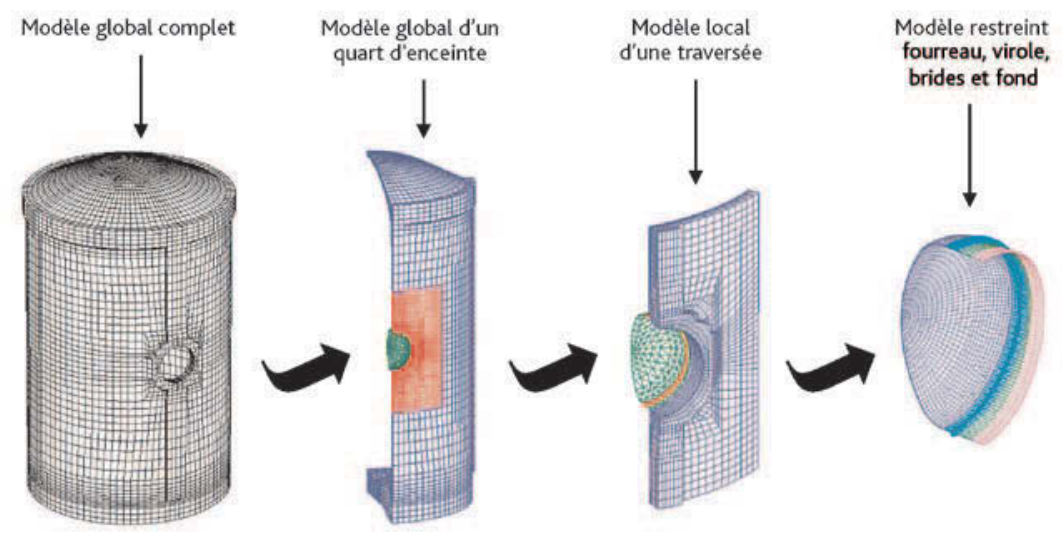

Figure 6.4. Modèles emboîtés retenus : modèle global complet, modèle global d'un quart d'enceinte, modèle local d'une traversée, modèle restreint fourreau/virole/brides/fond.
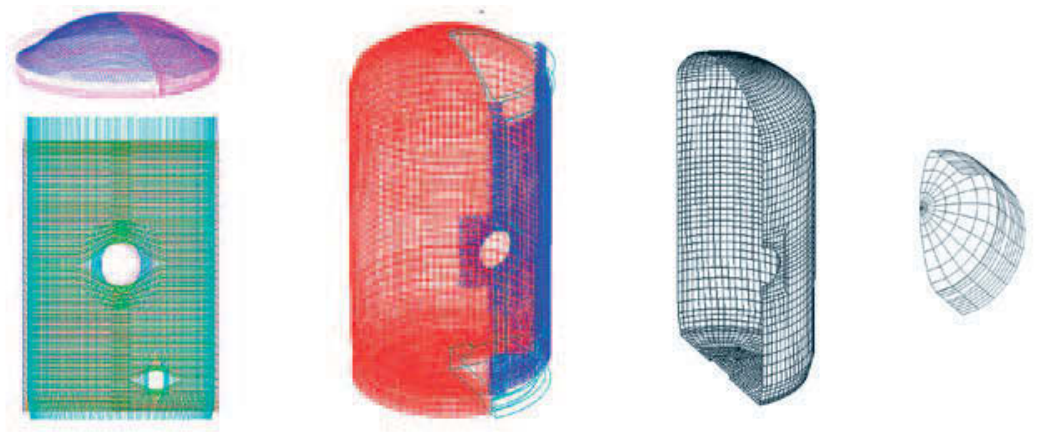

Figure 6.5. Maillage représentant les câbles de précontrainte, le ferraillage, la peau métallique, le tampon de l'accès des matériels (TAM) utilisés pour le modèle global complet. 
Le retrait et le fluage du béton sont estimés de façon réglementaire, suivant les formules du BPEL 1999, lors de la mise en tension des câbles, et pendant la durée de 30 ans. Ils sont introduits à chaque étape du calcul sous la forme d'un chargement de type « déformations initiales », en fonction des caractéristiques de séchage du béton, de l'âge du chargement et du champ des contraintes.

Le comportement différé de l'enceinte calculé par la méthode des éléments finis a été comparé aux résultats des mesures d'auscultation réalisées à 20 ans par EDF, ce qui a permis de recaler les paramètres des formules BPEL par rapport aux résultats des mesures.

\subsubsection{Modèle quart d'enceinte}

Le calcul thermomécanique du comportement de l'enceinte dans les conditions du scénario AF de fusion du cœur a été réalisé avec un maillage représentant un quart de cette enceinte afin de réduire les temps de calcul.

Le calcul de la précontrainte et du fluage, réalisé avec le modèle complet de l'enceinte, est utilisé pour le modèle quart d'enceinte, avant d'appliquer les chargements de pression et de température dus à l'accident de fusion du cœur.

Le modèle quart d'enceinte permet de représenter, de la même manière que le modèle complet, le béton de l'enceinte, les câbles de précontrainte, le ferraillage passif, la peau métallique, le béton de protection posé sur le radier, les structures internes, la virole de l'accès des matériels avec son fourreau, les brides et le fond. La figure 6.4 présente le maillage utilisé pour ce calcul.

Le béton est modélisé par des éléments finis massifs linéaires à huit nœuds avec une loi de comportement non linéaire prenant en compte une fissuration volumique homogène du béton (suivant un modèle rhéologique de fissuration fictive appelé « OTTOSEN ») ; les câbles de précontrainte ainsi que le ferraillage passif sont modélisés par des éléments finis de type barre à deux nœuds avec une loi de comportement non linéaire de type élastoplastique à écrouissage isotrope. La peau métallique est modélisée par des éléments finis de type coque avec une loi de comportement non linéaire de type élastoplastique à écrouissage isotrope. Le sol est simulé à l'aide d'un élément unique s'étendant au-delà de la surface horizontale du radier, ce qui permet un décollement de ce dernier en fonction des chargements dus à l'accident de fusion du cœur [4].

\subsection{Analyse des résultats des calculs réalisés à l'aide du modèle « quart d'enceinte »}

L'analyse des résultats des calculs pour l'accident de fusion du cœur étudié conduit aux remarques suivantes :

- les calculs confirment les emplacements des zones les plus sensibles de l'enceinte de confinement, notamment la zone du tampon d'accès des matériels (TAM) et la 
zone du gousset (figure 6.6) qui présente des fissurations traversantes en relation avec la galerie de précontrainte ;

- la comparaison des résultats des calculs pour le scénario AF et de ceux obtenus pour un scénario sans pic de pression P3 montre le peu d'effet de ce pic sur le comportement ultérieur de l'enceinte de confinement (les déplacements de l'enceinte sont quasi réversibles dans la zone courante durant le pic de pression) ;

- la stabilité d'ensemble de la structure est assurée par l'intégrité des câbles de précontrainte ;

- la déformation plastique équivalente maximale de la peau métallique obtenue juste après le pic de pression (instant P4 sur la figure 6.3) est plus importante que celle obtenue au pic de pression (instant P3 sur la figure 6.3) ;

- l'extrapolation du comportement mécanique de l'enceinte à d'autres scénarios est possible car les résultats des calculs mettent en évidence que les phénomènes mécaniques dépendent principalement de la pression ;

- les déchirures éventuelles de la peau métallique et les fissures dans la paroi en béton précontraint de l'enceinte constituent les chemins de fuite ;

- les déformations calculées de la peau métallique restent très inférieures à la valeur de la déformation à rupture de l'acier; d'après les résultats obtenus, il ne devrait pas y avoir de déchirure de la peau et l'étanchéité de l'enceinte de confinement devrait être conservée.

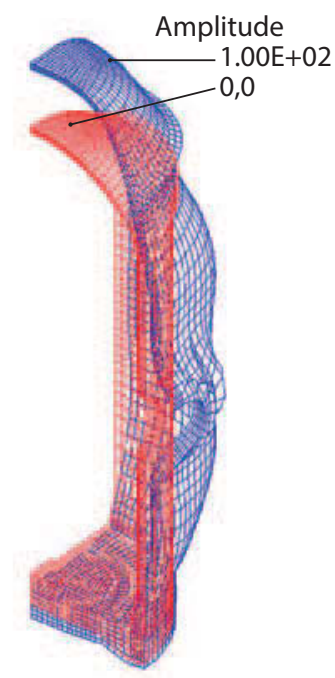

Scénario AF au temps : $9.06890 \mathrm{E}+04 \mathrm{sec}$

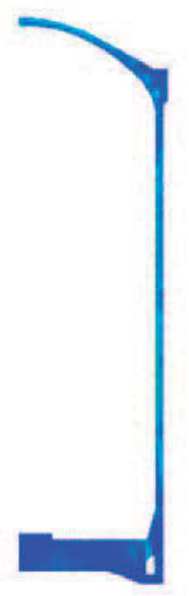

W1 dans un plan à $67,5^{\circ}$
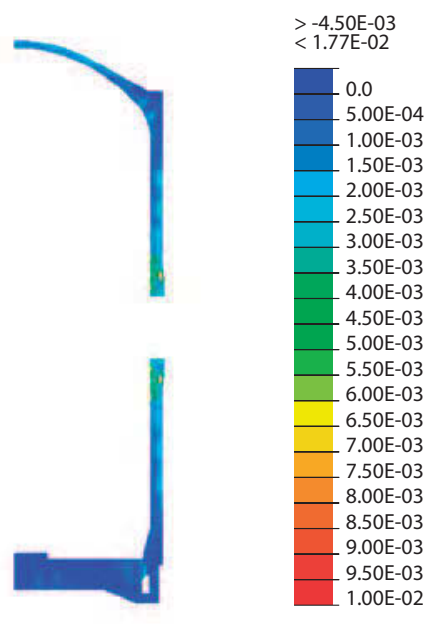

W1 dans un plan à $0^{\circ}$ au temps : $90689 \mathrm{sec}$

Figure 6.6. Déformée de l'enceinte amplifiée 100 fois et fissuration du béton, dans l'axe du TAM et en zone courante, au pic de pression du scénario AF (instant P3). 
L'analyse et l'interprétation des résultats des études précédentes ont nécessité, pour estimer les risques de défaillance de l'enceinte (peau métallique, paroi en béton précontraint), en fonction des déformations atteintes, le recours à des résultats expérimentaux afin de définir les critères d'acceptabilité des résultats des calculs non linéaires effectués ; à cet effet, les résultats des calculs ont été confrontés à quelques résultats expérimentaux obtenus sur des maquettes, notamment la maquette PCCV utilisée pour le programme américano-japonais (NUPEC - NRC - Sandia) décrit au paragraphe suivant. Un groupe d'experts a participé à cette confrontation calcul-expérience dans le but de définir ces critères.

\subsection{Analyse des résultats d'essais sur maquette}

Le recours à des résultats d'essais représentatifs du problème étudié est un élément important pour la validation des calculs réalisés avec le logiciel de calculs mécaniques CAST3M. La difficulté est de trouver des essais représentatifs pour les conditions de chargement considérées [5].

La maquette PCCV (NUPEC - NRC - Sandia) représente à l'échelle $1 / 4$ une enceinte de confinement en béton précontraint avec une peau métallique. Des essais de mise en pression de la maquette avec de l'air sec à température ambiante ont été réalisés par les laboratoires Sandia ; ils ont été suivis d'un essai destructif conduit jusqu'à la ruine de l'enceinte (comportement ultime).

Les essais réalisés avec la maquette PCCV ont montré l'existence de déchirures de la peau métallique conduisant à des fuites significatives pour des valeurs de pression de l'ordre de 10,7 bars absolus (2,5 fois la pression de dimensionnement de la maquette [6]).

L'analyse des résultats des essais réalisés avec cette maquette et de leurs simulations numériques a été effectuée dans le cadre de l'International Standard Problem (ISP48) organisé par l'OCDE, auquel l'IRSN a participé. Des calculs ont été réalisés avec le logiciel CAST3M en utilisant la même démarche que celle utilisée pour les calculs du projet EPS2 [6].

Les calculs réalisés par l'IRSN ainsi que par les différentes équipes qui ont participé à cette intercomparaison n'ont pas prévu les déchirures constatées à la pression de 10,7 bars absolus, même en tenant compte des différentes singularités géométriques [7].

Pour cette pression dans l'enceinte, la déformation circonférentielle mesurée de la peau métallique dans la zone courante du fût est de 0,17 \% et la déformation plastique équivalente calculée est de l'ordre de 0,3 à 0,5\%. Le même ordre de grandeur est obtenu par les calculs des déformations. Mais cette valeur est inférieure à la limite de la déformation à la rupture (de l'ordre de $10 \%$ ) obtenue lors des essais de caractérisation de la peau réalisés après les essais sur la maquette.

Pour pouvoir retrouver les déchirures de la peau métallique qui correspondent à un phénomène local, il faudrait que les modèles utilisés soient à l'échelle de ce phénomène en tenant compte des singularités constituées par les différentes soudures et les ancrages de la peau ainsi que par les fissures éventuellement créées dans le béton, 
en utilisant des outils qui permettent de simuler la localisation des déformations et la déchirure de la peau métallique.

C'est cette difficulté d'interprétation des résultats des essais réalisés avec la maquette PCCV qui a conduit l'IRSN à faire appel au groupe d'experts cité plus haut. Ce groupe avait pour mission de conseiller l'IRSN sur la façon d'utiliser au mieux les résultats des essais dans leur application aux calculs des enceintes de confinement réalisés dans le cadre du projet EPS2 de l'IRSN. À l'issue de ses travaux, le groupe a proposé le critère d'application suivant : la déformation plastique maximale dans la zone courante de la peau métallique obtenue par les calculs non linéaires dans la zone courante doit être inférieure à une valeur de 0,30\% $\%$ 0,15\%; au-delà de cette valeur, une déchirure de la peau métallique est très probable par effet de localisation des déformations. En effet, par cet effet de localisation, les déplacements se concentrent sur un point de la structure où la déformation va dépasser le critère de déchirure du matériau.

Une valeur de déformation de 0,3\% pour la peau métallique correspond à une pression dans l'enceinte de l'ordre de 10,5 bars absolus pour le scénario AF, ce qui conduit à considérer que la valeur de la pression limite conduisant à la défaillance du confinement est de l'ordre de 10 bars absolus (2 fois la pression de dimensionnement de l'enceinte de confinement).

Il convient de rappeler que la tenue ultime des traversées n'a pas été considérée dans cette étude.

\subsubsection{Modèle local de la traversée du tampon de l'accès des matériels}

La modélisation d'un quart d'enceinte, avec ses câbles de précontrainte et son ferraillage passif, et des lois de comportement mécanique non linéaires, nécessite un temps de calcul considérable, malgré une discrétisation spatiale relativement grossière de la géométrie. Pour étudier le comportement des zones sensibles telles que le tampon d'accès des matériels (TAM), notamment le risque d'écartement des brides constituant le système de fermeture du tampon (un tel écartement conduisant à une fuite directe dans l'atmosphère), une modélisation plus fine a donc été adoptée. Cette modélisation permet de représenter la géométrie exacte des brides et des boulons qui les maintiennent jointives. Ce modèle local comporte les mêmes éléments que le modèle global (béton, peau métallique, ferraillage passif et câbles de précontrainte composant une partie du fût de 10,60 m de large et 23,40 m de haut, virole du tampon d'accès, brides et boulons, goussets et collerettes d'ancrage de la virole dans le béton, etc.) ; les mêmes chargements thermomécaniques et les mêmes lois de comportement des matériaux sont appliqués à ce modèle local ainsi que la précontrainte, le retrait du béton et le fluage introduits dans le modèle global.

À partir d'un même calcul quart d'enceinte, plusieurs calculs ont été réalisés pour la traversée, en modifiant certains paramètres tels que les caractéristiques mécaniques des boulons, le ferraillage passif, le maillage, les conditions aux limites, le serrage des boulons. Ces études de sensibilité ont permis d'apprécier les incertitudes dues à la modélisation, aux calculs et aux matériaux, qui sont de l'ordre de $15 \%$. 
Les brides ont été modélisées par des éléments de coque, de même que la peau, les goussets et les collerettes et l'ensemble fourreau/virole/brides/fond. Les fers et les câbles ainsi que les 44 boulons de fermeture du TAM ont été modélisés par des éléments de type barre à deux nœuds (figure 6.7). Trois types de boulons ont été envisagés, les boulons utilisés initialement pour les enceintes des centrales REP de $900 \mathrm{MWe}$ (diamètre $33 \mathrm{~mm}$; limite élastique $238 \mathrm{MPa}$ ) et deux autres types de boulons jugés plus résistants (respectivement diamètre de $33 \mathrm{~mm}$ et limite élastique de $729 \mathrm{MPa}$; diamètre de $24 \mathrm{~mm}$ et limite élastique de $852 \mathrm{MPa}$ ).
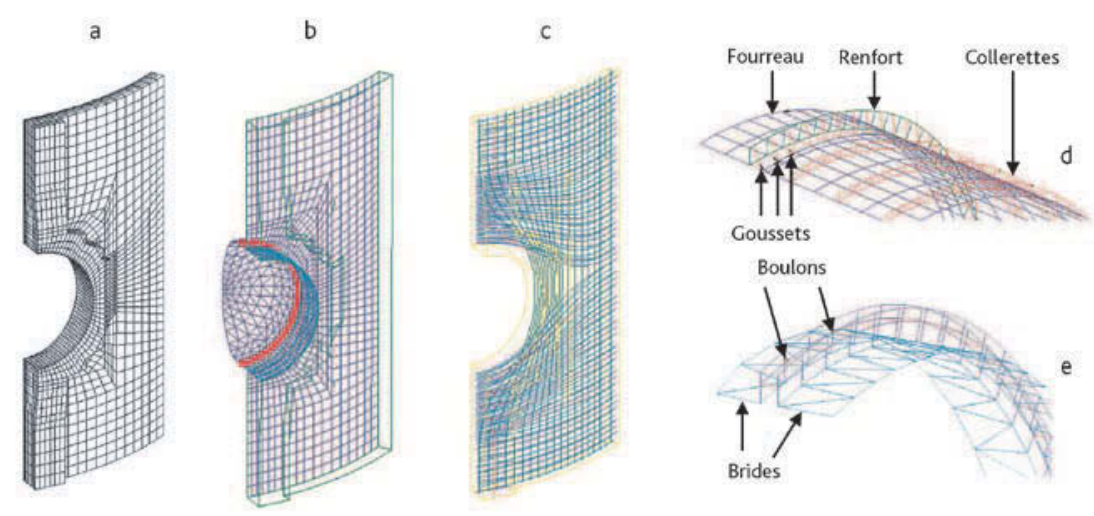

Traverse
7164 mds 5676 elts

Figure 6.7. Traversée en béton (a), peau/fourreau/brides/fond (b), câbles (c), détail fourreau (d), détail brides et boulons (e).

Les conditions aux limites du modèle local reprennent à chaque pas de temps les champs de déplacements, obtenus à l'aide du calcul réalisé avec le modèle quart d'enceinte, sur le contour du modèle local. La validité de cette méthode est vérifiée notamment par la comparaison des résultats obtenus avec des modèles locaux d'échelles différentes.

Faute de données expérimentales sur le comportement mécanique des joints vieillis, les études réalisées ne tiennent pas compte de la présence d'un joint d'étanchéité entre les deux brides, et ne fournissent comme résultat que l'écartement entre ces brides. Les principaux résultats obtenus sont les suivants :

- le choix des boulons (caractérisés par leur section et la limite élastique de l'acier) est le paramètre critique de l'étude mécanique, avec des répercussions considérables sur l'amplitude de l'écartement des brides ;

- le pic de pression et de température (instant P3) influence relativement peu la valeur de l'écartement en fonction de la pression ; l'écartement des brides est donc en grande partie conditionné par les mouvements d'ovalisation et de voilement de l'enceinte autour du fourreau de l'accès des matériels, peu sensibles à la température ; 
- quel que soit le scénario, la refermeture des brides n'est que partielle avec la chute de la pression; ceci est dû à la plastification des boulons et à l'absence de réversibilité des déformations de l'enceinte en béton autour de la virole du TAM;

- l'écartement des brides est à peu près constant le long de la circonférence du fourreau avec une longueur d'ouverture de l'ordre de $4 \mathrm{~m}$ (pour la demicirconférence) ; la section de fuite est quasiment proportionnelle à l'écartement maximal des brides.

\subsubsection{Modèle restreint}

La modélisation de la liaison entre les brides constitue un aspect délicat de l'étude thermomécanique, et le plus sensible a priori en termes d'écartement des brides. Dans le modèle local, le choix des éléments finis de type coque conduit à surévaluer les ouvertures des brides. C'est la raison qui a motivé la réalisation d'un modèle restreint ; ses principales caractéristiques sont les suivantes :

- des éléments massifs sont utilisés pour modéliser le fourreau métallique, les brides, les boulons et le fond hémisphérique du tampon au lieu d'éléments de type coque ou poutre, afin de s'affranchir des difficultés liées à l'utilisation d'éléments de ce type pour la définition des conditions aux limites;

- le maillage est beaucoup plus fin et permet de reproduire de façon plus fidèle la géométrie réelle (changements d'épaisseur, chanfreins des soudures, etc.) ;

- les goussets, les collerettes, le béton et le ferraillage passif ne sont plus représentés dans le modèle ; il est supposé que le béton impose ses déplacements et ses déformations aux parties métalliques de rigidités plus faibles.

Les lois de comportement utilisées sont identiques à celles utilisées pour le calcul de la traversée. Par raison de symétrie, le maillage réalisé représente une demi-circonférence (figures 6.8 et 6.9).

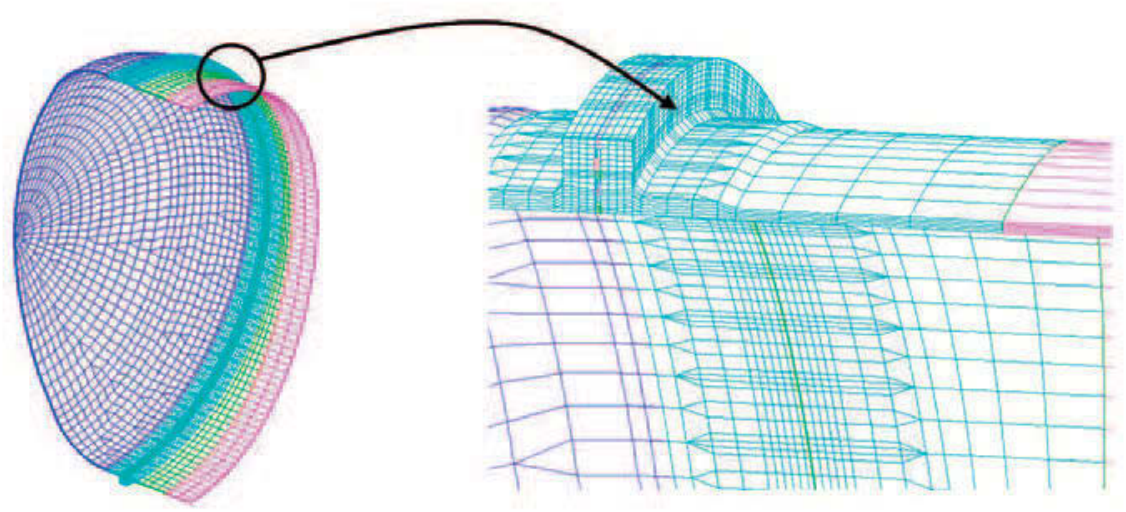

Figure 6.8. Maillage de l'ensemble du fourreau et de la bride du côté du fond du tampon (avec cales et boulons). 

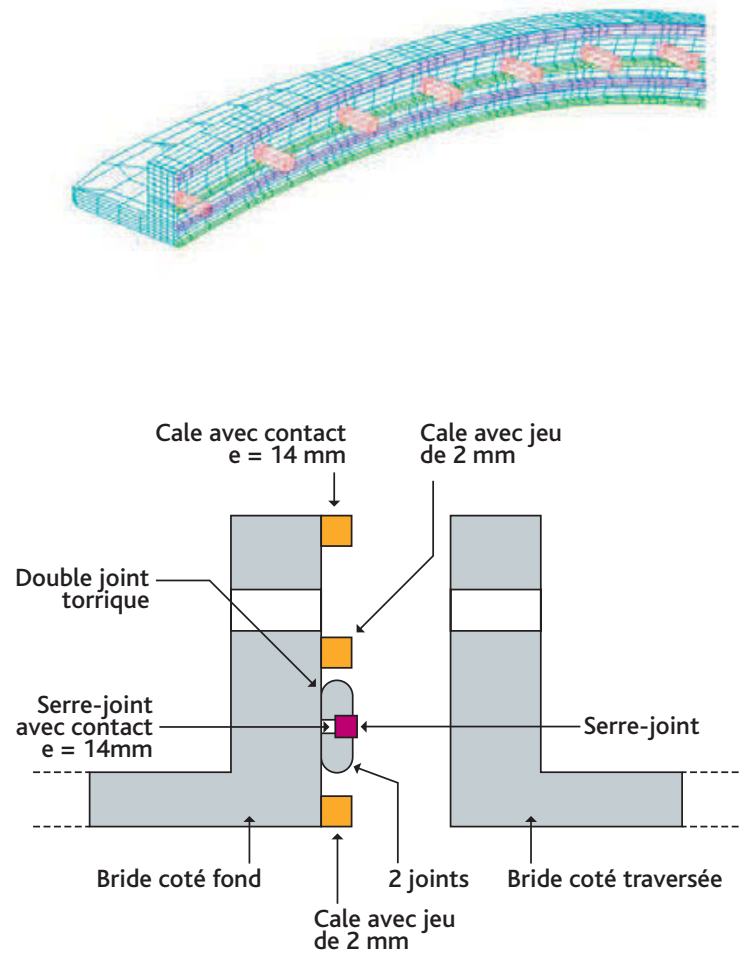

Figure 6.9. Maillage d'une bride (avec cales, serre-joint et boulons) et schéma en coupes des brides.

La mise en œuvre du modèle restreint est faite de façon similaire à celle du modèle local. Les conditions aux limites en déplacements imposés résultent du calcul avec le modèle local ; elles sont appliquées au contour du modèle restreint. Le modèle restreint avec ses éléments massifs et sa représentation plus précise des brides, cales et serre-joints permet d'améliorer la compréhension du comportement du système de fermeture. Notamment, il a permis de mettre en évidence l'effet prépondérant des sollicitations de cisaillement des boulons jusqu'à leur rupture, pour des pressions modérées dans l'enceinte de confinement, avec un risque important de perte d'étanchéité de la fermeture du tampon.

Ce modèle a ainsi montré :

- la complexité des mécanismes de déformation des brides, excluant toute possibilité d'extrapolation à partir de modèles simplifiés, et le fort couplage entre les phénomènes d'ovalisation et de voilement des brides ;

- le faible effet des sollicitations axisymétriques du béton, par l'intermédiaire du fourreau, sur l'écartement des brides et le cisaillement des boulons (chargements dus à la pression dans l'enceinte de confinement, pincement du fourreau sous l'effet de la précontrainte de compression imposée dans le béton par la mise sous tension des câbles) et l'importance des déformations non axisymétriques 
imposées par l'enceinte de confinement au fourreau, responsables de l'ovalisation et du voilement des brides ;

- l'importance du choix des boulons (notamment leur section et la limite élastique de leur acier) sollicités en cisaillement ;

- l'influence du jeu entre boulons et brides.

Pour ce qui concerne ce dernier point notamment, les résultats ont montré qu'en l'absence de prise en compte du jeu entre brides et boulons, dans le cas où les deux brides sont libres de glisser l'une par rapport à l'autre, la plastification des boulons a lieu pour des pressions basses (entre 3,2 et 5,5 bars absolus suivant les boulons choisis). Ces valeurs basses, dues au cisaillement des boulons, sont fortement sensibles aux jeux entre boulons et brides.

La marge apportée au cisaillement par un jeu de l'ordre de $3 \mathrm{~mm}$, jeu acceptable à l'égard du risque de plastification des boulons, conduit, lors d'une combustion d'hydrogène, à un écartement irréversible des brides à partir de pressions comprises entre 6,2 et 8,5 bars absolus suivant les boulons choisis. Il apparaît qu'une réduction de la section des boulons est préjudiciable à leur résistance en traction, tandis qu'une augmentation de la limite élastique de l'acier est bénéfique à leur tenue mécanique au cisaillement.

\subsubsection{Comportement mécanique des enceintes des centrales REP de $1300 \mathrm{MWe}$}

\subsubsection{Démarche utilisée dans les études mécaniques}

L'objectif poursuivi pour ce type d'enceinte est similaire à celui des études mécaniques réalisées pour une enceinte d'un REP de $900 \mathrm{MWe}$. Il s'agit de déterminer la limite d'étanchéité de l'enceinte de confinement sous les chargements mécaniques et thermiques auxquels est soumise cette enceinte lors d'un accident de fusion du cœur. Cependant la démarche utilisée est différente pour deux raisons essentielles :

- la paroi interne de l'enceinte interne n'a pas de revêtement métallique ; les études mécaniques visent donc à déterminer la capacité du béton précontraint de l'enceinte interne à maintenir un confinement sous différents chargements accidentels ;

- les fuites de l'enceinte interne se retrouvent dans l'espace entre enceintes ; cet espace est normalement en dépression et les gaz et les aérosols extraits par le système de mise en dépression de cet espace (EDE) sont filtrés avant rejet ; les études mécaniques précédentes doivent donc être prolongées par un examen des défaillances possibles de ce système et de l'enceinte externe en situation d'accident de fusion du cœur, en tenant compte de conditions climatiques sévères (notamment vents de vitesse élevée).

Des études relatives à ces enceintes sont menées à l'IRSN en support de son EPS de niveau 2 des centrales REP de 1300 MWe. Ces études viennent également en appui de l'expertise des dossiers d'EDF en vue des troisièmes visites décennales (VD3) des 
centrales REP de 1300 MWe, notamment les dossiers relatifs au traitement des accidents de fusion du cœur, sachant qu'EDF développe ses propres études sur le sujet. Une des difficultés particulières, qui apparaît dans les quantifications de l'EPS de niveau 2 ou lors des discussions techniques avec l'exploitant, est l'évaluation des fuites à travers la paroi en béton précontraint de l'enceinte interne sous pression, évaluation qui conditionne l'ampleur des rejets de substances radioactives dans l'espace entre enceintes et la possibilité du système EDE à maintenir cet espace en dépression. Des réponses à des questions difficiles sont nécessaires pour obtenir cette quantification, notamment :

- l'appréciation de la fissuration du béton (espacement, ouverture, longueur des fissures, etc.) à partir des états des contraintes mécaniques dans les parois de l'enceinte; les formules réglementaires applicables au retrait et au fluage du béton fournissent en effet des valeurs numériques qui sont très éloignées des valeurs mesurées ; EDF a, sur ce sujet, initié un projet national d'étude (le projet CEOS) auquel l'IRSN participe ; les résultats des actions de recherche menées dans le cadre de ce projet sont prévus pour 2013 ;

- la quantification des fuites à travers un réseau de fissures qui communiquent entre elles sans que chacune d'elles soit obligatoirement traversante ; cette question est étudiée à l'IRSN dans le cadre du projet ECOBA (étude du confinement dans les ouvrages de béton armé) financé par l'Agence nationale de la recherche (ANR) ; les résultats des actions de recherche menées dans le cadre de ce projet sont prévus pour 2014.

Compte tenu des difficultés indiquées ci-dessus et dans l'attente des résultats des projets précités, la démarche utilisée dans les études IRSN est semi-empirique. Les étapes de cette démarche peuvent se résumer comme suit :

- le degré de fissuration de la paroi de l'enceinte interne est calculé, à l'aide du logiciel CAST3M, à partir d'un modèle aux éléments finis de cette paroi, en fonction de la précontrainte dans les câbles, du fluage et du retrait du béton ;

- les résultats de ces calculs sont ajustés aux résultats expérimentaux relevés lors des épreuves des enceintes à leur pression de dimensionnement (des mesures de fissuration du béton sont notamment réalisées à la suite de ces épreuves) ;

- le modèle ainsi ajusté est ensuite validé en confrontant les résultats à ceux d'expériences réalisées sur des maquettes dans des conditions approchant celles d'un accident de fusion du cœur (voir la description des essais MAEVA au § 6.3.3.2) ;

- le modèle de calcul, réalisé avec le logiciel CAST3M, ainsi validé est finalement appliqué à l'enceinte interne d'un REP de 1300 MWe supposée soumise aux chargements de pression et de température d'un accident de fusion du cœur jugé suffisamment enveloppe.

L'achèvement de ces différentes étapes devrait permettre d'obtenir en 2013 des estimations des rejets dans l'espace entre enceintes.

Il est à noter que le retour d'expérience des épreuves réalisées à la pression de dimensionnement et les résultats des premiers calculs du comportement mécanique des 
enceintes des centrales REP de 1300 MWe montrent que le béton de l'enceinte interne ne demeure pas partout en compression mais présente des zones en traction susceptibles de se fissurer. Par ailleurs, compte tenu des déformations différées du béton précontraint, la précontrainte diminue dans le temps, ce qui augmente l'étendue des zones pouvant se trouver en traction. Les zones concernées (ainsi que celles où ont été constatés, lors des épreuves en pression, des défauts de bétonnage) ont été recouvertes d'un revêtement qui permet de retrouver une étanchéité conforme aux exigences requises en termes de taux de fuite.

L'étude des défaillances possibles du système EDE dans les conditions d'un accident de fusion du cœur est effectuée en parallèle à l'IRSN dans le cadre des études en support à son EPS de niveau 2. Il s'agit de déterminer les limites de fonctionnement du système de ventilation (c'est-à-dire sa capacité à maintenir l'espace entre enceintes en dépression), de son système de filtration et des pièges à iode très sensibles au taux d'humidité.

\subsubsection{Expériences effectuées en support des études mécaniques des enceintes REP de1 300 MWe : les essais MAEVA}

En 1994, EDF a décidé de réaliser une maquette d'enceinte de confinement afin d'étudier la résistance mécanique et d'évaluer l'étanchéité des enceintes de confinement des réacteurs français. La maquette réalisée représente une zone courante de l'enceinte interne à l'échelle 1/3 pour le diamètre et l'échelle 1/1 pour l'épaisseur du mur. L'espace entre enceintes est également représenté mais l'enceinte externe en béton est remplacée sur la maquette par une paroi métallique (figure 6.10). L'objectif visé par EDF était d'effectuer une étude expérimentale du comportement thermomécanique de l'enceinte interne en béton précontraint pour des situations de dimensionnement et des situations au-delà du dimensionnement.

Plus précisément, les principaux objectifs des essais étaient les suivants :

- l'étude de la propagation de la chaleur dans une paroi en béton précontraint en présence d'un mélange d'air et de vapeur ;

- l'étude du comportement d'une paroi en béton précontraint au-delà des situations de dimensionnement, en soumettant la maquette à des scénarios de montée en pression et en température ;

- l'évaluation des taux de fuite d'air et de vapeur dans les conditions d'un accident par comparaison à ceux mesurés avec de l'air sec lors des épreuves ;

- l'étude du comportement de revêtements en matériaux composites pour les séquences d'épreuve et les différents scénarios accidentels ainsi que la validation de leurs conditions de mise en œuvre à l'échelle industrielle.

Les résultats devaient permettre d'estimer la capacité de l'enceinte interne à assurer la fonction de confinement dans les conditions accidentelles de dimensionnement et audelà de ces conditions ainsi que de préciser les marges sur les taux de fuite par rapport aux conditions des épreuves. 
La maquette MAEVA était constituée d'une paroi cylindrique en béton précontraint de $16 \mathrm{~m}$ de diamètre, 1,2 $\mathrm{m}$ d'épaisseur et $5 \mathrm{~m}$ de hauteur (figure 6.10) ; elle a été réalisée sur le site de Civaux, avec du béton de mêmes caractéristiques que celui utilisé pour l'enceinte de confinement de la tranche 2 de la centrale de Civaux (béton à hautes performances). La dalle supérieure était soutenue par quatre poteaux en béton précontraint disposés dans chacun des quarts de la surface de cette dalle. La paroi interne était divisée en quadrants dont deux étaient revêtus d'une peau d'étanchéité en matériau composite similaire à celui utilisé pour les réparations des tranches en exploitation.

La précontrainte a été calculée de façon à obtenir, comme pour les enceintes des tranches en exploitation, une compression résiduelle moyenne de $1 \mathrm{MPa}$ à une pression de 6,5 bars absolus.

Plusieurs essais ont été réalisés avec cette maquette, avec de l'air et avec un mélange d'air et de vapeur, jusqu'à la pression de dimensionnement de la maquette $(5,5$ bars relatifs) ; pour le domaine des accidents de fusion du cœur, un essai avec de l'air a été réalisé à une pression de 9,75 bars relatifs (figure 6.11).

Lors de chaque essai, les mesures effectuées ont visé à déterminer les fuites dans l'espace annulaire, divisé en quatre quarts étanches (chaque quart étant appelé caisson), avec de l'air sec et avec un mélange d'air et de vapeur, les températures dans l'enceinte et dans la paroi en béton, la pression dans l'enceinte et les déplacements de la paroi interne de la maquette. De plus, un relevé des fissures, une auscultation visuelle des revêtements en matériau composite et des essais d'arrachement de ces revêtements ont

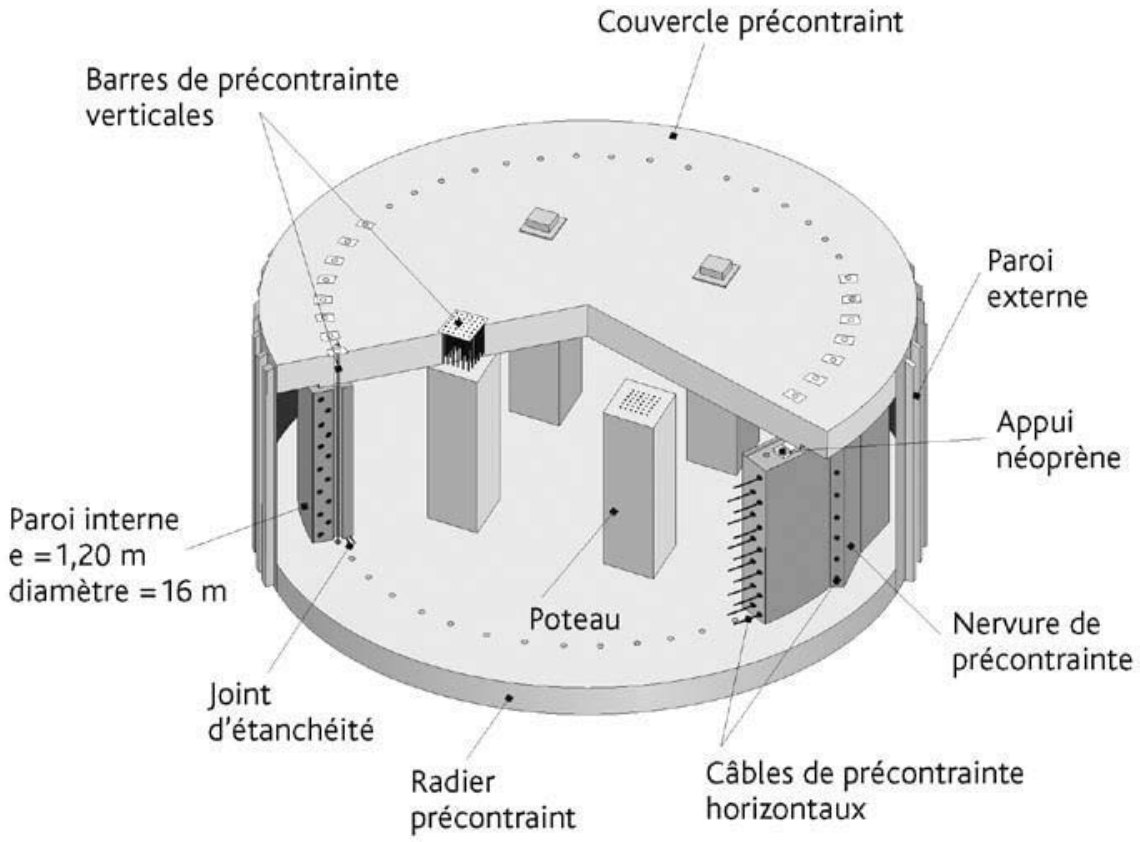

Figure 6.10. Schéma de la maquette MAEVA. 


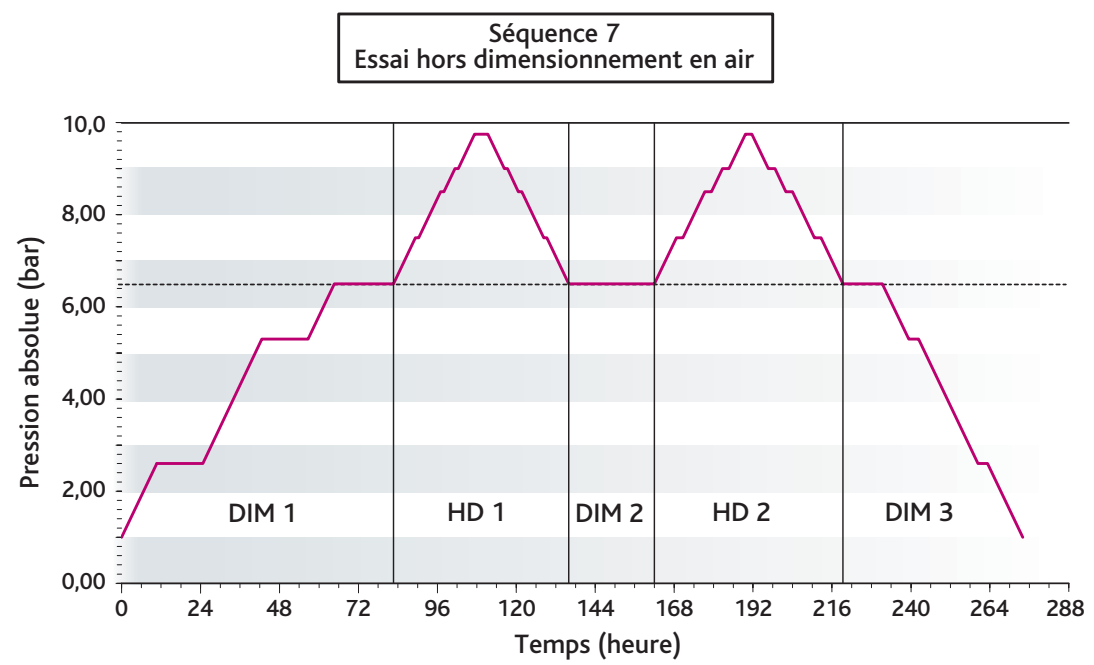

Figure 6.11. Évolution de la pression lors de l'essai réalisé sur la maquette MAEVA dans le domaine des accidents de fusion du cœur.

été réalisés. Ces derniers tests ont permis de quantifier l'adhérence des revêtements sur la paroi en béton après les séquences d'essais.

L'analyse des résultats a également permis de déterminer, d'une part le coefficient de transposition à retenir entre le taux de fuite pour de l'air sec et le taux de fuite pour un mélange d'air et de vapeur, ce coefficient étant ensuite utilisé pour déterminer les taux de fuite des enceintes réelles en situation accidentelle à partir des taux de fuite mesurés lors des épreuves, d'autre part l'évolution de la fissuration et des fuites mesurées pour plusieurs scénarios accidentels. Ce sont notamment ces derniers résultats sur l'évolution de la fissuration et les mesures des fuites qui sont exploités pour valider la méthode de quantification des fuites décrite au paragraphe 6.3.3.1 (projet national CEOS initié par EDF et projet ECOBA financé par l'ANR, projets en cours au moment de la rédaction du présent ouvrage).

\subsubsection{Bilan et perspectives concernant les études effectuées à I'IRSN au sujet du comportement mécanique des enceintes de confinement en situation d'accident de fusion du cœur}

\subsubsection{Bilan et perspectives pour les enceintes des centrales REP de $900 \mathrm{MWe}$}

Les calculs non linéaires réalisés à l'IRSN ont permis de déterminer le comportement mécanique des enceintes de confinement des réacteurs de $900 \mathrm{MWe}$ dans les conditions d'un accident de fusion du cœur et, plus spécifiquement, celui des zones sensibles de ces ouvrages. L'étanchéité de ces enceintes est assurée par la peau métallique présente sur le parement interne de l'enceinte. Le recours à des résultats d'essais sur maquettes 
a permis, en tenant compte du retour d'expérience, de définir un critère de perte d'étanchéité de la peau métallique adapté aux calculs par la méthode des éléments finis.

La valeur retenue pour la pression conduisant à la perte d'étanchéité d'une telle enceinte est de l'ordre de 10 bars absolus ( 2 fois la pression de dimensionnement). Il convient toutefois de noter que les calculs ont été effectués pour une peau idéale, sans tenir compte des défauts pouvant exister dans les soudures ou d'une dégradation éventuelle par la corrosion, car il est très difficile de simuler numériquement ces phénomènes. Pour cette raison, un coefficient de sécurité devrait être affecté à la pression précédente, en fonction du degré de connaissances des phénomènes précités, afin de déterminer la pression limite d'étanchéité d'une telle enceinte.

Les résultats peuvent être extrapolés aux scénarios d'accident de fusion du cœur autres que le scénario AF. Cette extrapolation est possible du fait que les phénomènes mécaniques dépendent principalement de la pression.

Pour ce qui concerne l'étanchéité du TAM, les modèles local et restreint ont mis en évidence deux modes de perte du confinement, correspondant à une rupture en traction et à une rupture par cisaillement des boulons. Ces deux modes de rupture présentent des effets de seuils dépendant du choix des boulons et des conditions spécifiques de chaque installation (jeu initial entre le corps du boulon et l'alésage de la bride, état de surface des brides et frottements, etc.). En tout état de cause, quel que soit le mode de rupture, ces études ont confirmé la vulnérabilité du système de fermeture des brides retenu à la conception, dans les conditions d'un accident de fusion du cœur. EDF a décidé de changer les boulons (changement de nuance et de diamètre) du TAM de telle sorte que l'enceinte résiste à une pression accidentelle d'au moins 8 bars absolus.

En dehors du TAM, le comportement des autres zones sensibles de l'enceinte, comme les différentes traversées et pénétrations, doit aussi être apprécié pour les conditions d'un accident de fusion du cœur, car la pression limite d'étanchéité de l'enceinte dépend également de ce comportement. Des actions sont menées par EDF à ce sujet dans le cadre des réexamens de sûreté concernant la vérification du comportement des matériels en conditions d'accident grave.

\subsubsection{Bilan et perspectives pour les enceintes des centrales REP de 1300 MWe}

Dans le cadre de la réalisation par l'IRSN d'une EPS de niveau 2 pour les centrales REP de $1300 \mathrm{MWe}$, celui-ci a établi une démarche d'étude du comportement des enceintes à double parois ; elle sera utilisée en 2013 lors des discussions relatives au réexamen de sûreté associé aux troisièmes visites décennales des centrales REP de $1300 \mathrm{MWe}$.

Cependant, cette démarche, fondée sur les résultats des épreuves des enceintes et des essais réalisés avec la maquette MAEVA, présente des incertitudes importantes car la fissuration du béton est difficile à caractériser et la quantification des fuites à travers les fissures est donc imprécise. 
L'amélioration des connaissances dans ces deux domaines fait l'objet de deux projets dont les résultats sont attendus pour 2013 et 2014 :

- le projet national CEOS, piloté par l'Institut pour la recherche appliquée et l'expérimentation en génie civil (IREX), d'une durée de 4 ans; les objectifs de ce projet, auquel l'IRSN participe, sont l'étude de la fissuration de voiles ou de coques en béton sous différents chargements (chargement statique monotone, comportement au jeune âge sur une durée de dix jours après la mise en œuvre du béton, effet de déformations gênées, fissurations dues à un séisme) ;

- le projet ECOBA, financé par l'Agence nationale de la recherche (ANR, programme blanc) d'une durée de trois ans; l'objectif de ce projet est de mettre au point un outil fiable capable d'évaluer les fuites à travers une paroi en béton armé ou en béton précontraint dans les conditions d'un chargement sévère de pression éventuellement cumulé avec un chargement thermique.

Ces deux projets, qui sont complémentaires dans leurs objectifs, comprennent un volet modélisation et un volet expérimentation. EDF et le CEA sont parmi les maîtres d'ouvrage du projet CEOS. L'IRSN participe aux deux projets.

\subsection{Bipasses de l'enceinte de confinement}

\subsubsection{Introduction}

Les termes « bipasse du confinement » ou « contournement de l'enceinte » couvrent les situations qui, à la suite de dégradations d'équipements, entraînent une mise en communication directe du fluide primaire avec l'extérieur de l'enceinte de confinement (l'intérieur de bâtiments périphériques, ou directement l'environnement).

Un bipasse du confinement peut se produire lors du fonctionnement normal du réacteur, mais aussi lors d'un accident de type accident de dimensionnement (notamment lors d'un accident de rupture de tubes d'un générateur de vapeur) ou encore lors d'un accident avec fusion du cœur.

Les situations de fusion du cœur avec bipasse de l'enceinte de confinement font partie des situations qu'il faut viser à « pratiquement éliminer » car elles peuvent entraîner des rejets importants et précoces. En règle générale, ces situations n'ont pas été traitées à la conception pour les réacteurs en exploitation, car elles résultent de défaillances multiples ou présentent une probabilité d'occurrence très faible.

En revanche, pour le réacteur EPR, les situations de bipasse de l'enceinte de confinement sont traitées à la conception par des dispositions de conception et d'exploitation adaptées. Ces dispositions visent à prévenir les défaillances des circuits secondaires en cas de ruptures de tubes d'un générateur de vapeur, des circuits connectés au circuit primaire et qui sortent de l'enceinte de confinement ainsi qu'à assurer un confinement fiable dans ces situations (confinement qui permet de respecter dans tous les cas les objectifs fixés pour le réacteur EPR en termes de limitation des rejets). Peuvent 
être citées comme dispositions de prévention contribuant à l'élimination pratique des séquences accidentelles avec bipasse du confinement pour le réacteur EPR :

- les dispositions de conception du circuit d'injection de sécurité à basse pression (circuit ISBP/RRA, connecté au circuit primaire notamment dans certaines situations d'accident de perte de réfrigérant primaire ou lorsque le réacteur est à l'arrêt), qui est équipé sur les lignes d'injection à l'extérieur de l'enceinte de confinement de vannes d'isolement motorisées aptes à arrêter tout débit de fluide provenant du circuit primaire (en cas de défaillance des organes d'isolement [trois clapets antiretour] placés en amont dans l'enceinte de confinement). Ainsi les vannes sont dimensionnées pour conserver leur intégrité et assurer leur fonction d'isolement pour les pressions et températures du circuit primaire et pour des mélanges diphasiques eau-vapeur ;

- les dispositions de conception du circuit d'injection de sécurité à moyenne pression (circuit ISMP, connecté au circuit primaire notamment dans certaines situations d'accident de perte de réfrigérant primaire) similaires dans le principe à celles mises en place pour le circuit ISBP/RRA ;

- les dispositions de conception prises pour détecter et isoler les brèches des barrières thermiques des groupes motopompes primaires ;

- les dispositions de conception et de conduite mises en place pour les situations de ruptures de tubes d'un générateur de vapeur en vue de réduire les rejets à l'extérieur de l'enceinte de confinement :

- l'ISMP est dimensionnée avec une pression de refoulement suffisamment basse pour éviter que les soupapes et les vannes de décharge du circuit secondaire du générateur de vapeur concerné ne puissent être sollicitées avec un débit diphasique eau-vapeur et être endommagées ;

- en cas de niveau d'eau élevé du côté secondaire, le circuit RCV est automatiquement arrêté pour les mêmes raisons ;

- pour les états d'arrêt du réacteur, les dispositions de conception et de conduite relatives aux systèmes d'isolement de l'enceinte qui doivent être mises en œuvre pour assurer que l'enceinte de confinement sera fermée avant tout relâchement significatif de substances radioactives à l'intérieur de celle-ci ; cette exigence concerne notamment le tampon d'accès des matériels.

L'élimination pratique des situations de bipasse du confinement avec fusion du cœur suppose un examen systématique de toutes les séquences de bipasse envisageables, avec une analyse déterministe des lignes de défense correspondantes, complétée par les résultats des études probabilistes de sûreté, en tenant compte des incertitudes dues aux connaissances limitées de certains phénomènes physiques. Comme il est indiqué dans les directives techniques pour la conception et la construction de la prochaine génération de réacteurs nucléaires à eau sous pression, l'élimination pratique d'une séquence accidentelle ne peut pas reposer uniquement sur le respect d'une valeur de coupure probabiliste générique. Cela s'applique aux séquences de bipasse de l'enceinte de confinement, ce d'autant plus que les évaluations des probabilités de telles séquences associées à des rejets 
précoces importants dans l'environnement dépendent, en règle générale, largement de l'estimation des fréquences des modes de défaillance des équipements participant au confinement envisageables, pour certains déjà rencontrés lors de l'exploitation des réacteurs (fuite d'un clapet antiretour, défaillance d'une vanne d'isolement, défaillance d'une tuyauterie d'un circuit connecté au circuit primaire à l'extérieur de l'enceinte de confinement, rupture de la barrière thermique d'un groupe motopompe primaire, rupture d'un ou plusieurs tubes d'un générateur de vapeur, etc.), pour d'autres non rencontrés dans l'expérience d'exploitation. De ce fait, les incertitudes relatives aux données de fiabilité des différents circuits et systèmes pouvant par leur défaillance conduire à un bipasse du confinement doivent être évaluées et prises en compte lors de l'utilisation des résultats des études probabilistes de sûreté.

\subsubsection{Possibilités de bipasses de l'enceinte de confinement}

Lors du fonctionnement normal du réacteur, une défaillance de l'isolement entre le circuit primaire et l'un des circuits connectés au circuit primaire (notamment les circuits RIS, RRA, RCV, RRI pour les réacteurs en exploitation) peut entraîner une rupture à l'extérieur de l'enceinte de confinement du circuit concerné. Ces circuits ne sont en effet pas dimensionnés pour résister aux sollicitations résultant de l'arrivée de fluide primaire (mélange eau-vapeur à température et pression élevées) à la suite de la défaillance de l'isolement (défaillance d'un clapet ou d'une vanne d'isolement pour les circuits RIS, RRA, RCV, défaillance d'une barrière thermique d'un groupe motopompe primaire pour le circuit RRI). Outre la possibilité d'une rupture d'une tuyauterie à l'extérieur de l'enceinte de confinement, les échangeurs thermiques utilisés pour le refroidissement des circuits RRA, RCV et RRI constituent des points de fragilité mécanique ; des possibilités de fuite sont donc envisagées.

Au cours d'un accident de dimensionnement de type rupture de tubes d'un générateur de vapeur ou de type accident de perte de réfrigérant primaire, un bipasse de l'enceinte de confinement peut se produire dans les cas suivants :

- le cumul d'une rupture d'une tuyauterie de vapeur (RTV) et d'une rupture de tubes d'un générateur de vapeur (RTGV);

- le blocage en position ouverte d'une vanne du circuit de décharge à l'atmosphère (GCT-atmosphère) ou d'une soupape d'un générateur de vapeur, après la rupture de tubes d'un générateur de vapeur ;

- la rupture, dans un bâtiment périphérique, d'un circuit constituant une « extension de la troisième barrière du confinement » $(\S 6.1)$ (dont le système d'injection de sécurité [RIS] et le système d'aspersion dans l'enceinte [EAS]) en situation d'accident de perte de réfrigérant primaire.

Enfin, au cours d'un accident avec fusion du cœur, les bipasses de l'enceinte de confinement suivants peuvent se produire :

- une rupture simple ou multiple de tubes de générateurs de vapeur (RTGV) induite par une montée en pression du circuit primaire lors d'un renvoi d'eau sur le cœur en fusion ; 
- une rupture du circuit d'injection de sécurité due à une fuite affectant les organes d'isolement équipant les lignes d'injection de sécurité ;

- une rupture d'un circuit faisant partie des extensions de la troisième barrière de confinement (rupture d'un des circuits raccordés au circuit primaire ou rupture d'une barrière thermique d'un groupe motopompe primaire).

Les paragraphes suivants présentent quelques exemples de bipasses du confinement.

\subsubsection{Bipasses de l'enceinte de confinement pouvant survenir lors du fonctionnement du réacteur}

\subsubsection{Rupture du système d'injection de sécurité (RIS) à la suite d'une fuite affectant les organes d'isolement équipant les lignes d'injection d'eau dans le circuit primaire}

En cas de défaut d'étanchéité d'organes d'isolement (clapets) situés entre la partie « basse pression » du circuit RIS et le circuit primaire, une rupture de la partie à basse pression du circuit RIS (ISBP) peut se produire par mise en pression de cette partie si la fuite dépasse la capacité des soupapes de protection du circuit RIS. Une telle rupture provoque la vidange d'eau du circuit primaire à l'extérieur de l'enceinte de confinement. Selon les modes de défaillances des clapets, le débit calculé de vidange du circuit primaire varie entre $25 \mathrm{~m}^{3} / \mathrm{h}$ et $1000 \mathrm{~m}^{3} / \mathrm{h}$.

Les fréquences estimées de ces séquences accidentelles, dites de type $V$, dépendent du nombre d'organes d'isolement et des probabilités de défaillance y compris de cause commune retenues pour ces organes. Ces probabilités de défaillance de cause commune des différents clapets assurant l'isolement du circuit primaire étant faibles, la probabilité de fusion du cœur avec bipasse de l'enceinte de confinement est estimée, en considérant trois clapets dont deux sont diversifiés, à environ $10^{-8}$ par année.réacteur pour les réacteurs de $900 \mathrm{MWe}$. Cette valeur présente néanmoins une forte incertitude.

\subsubsection{Rupture du circuit de réfrigération intermédiaire (RRI) à l'extérieur de l'enceinte de confinement à la suite d'une rupture de la barrière thermique d'un groupe motopompe primaire}

Les barrières thermiques des pompes primaires sont refroidies par des serpentins faisant partie du système de réfrigération intermédiaire (RRI). La rupture d'un tel serpentin peut entraîner la rupture des tronçons du circuit RRI non dimensionnés à la pression du circuit primaire. Ceci conduit à une brèche non isolable du circuit primaire. La brèche peut, comme développé ci-après, intervenir dans l'enceinte de confinement ou à l'extérieur de celle-ci, conduisant dans ce dernier cas à un bipasse de cette enceinte.

Pour les tranches de $900 \mathrm{MWe}$, le refroidissement de chaque pompe primaire est assuré par une portion du système RRI traversant l'enceinte de confinement. À l'intérieur de l'enceinte, l'isolement de chaque ligne de refroidissement (une ligne pour chaque pompe primaire) repose sur la fermeture d'un clapet antiretour situé sur le circuit RRI en 
amont de la pompe primaire et sur la fermeture d'une vanne pneumatique située sur le circuit RRI en aval de la pompe primaire. La fermeture de la vanne est commandée automatiquement en cas de débit trop élevé dans le circuit RRI à la sortie de la barrière thermique de la pompe primaire (un débit trop élevé peut survenir à la suite d'une rupture d'un serpentin conduisant à une fuite de fluide primaire dans le circuit RRI). Le tronçon compris entre le clapet antiretour et la vanne résiste mécaniquement aux sollicitations de pression et de température associées à une fuite de fluide primaire dans le circuit RRI comme l'ont montré les calculs mécaniques réalisés par l'IRSN à cet effet. En revanche, en cas de défaillance du clapet antiretour ou de la vanne d'isolement, qui ne sont pas dimensionnés pour assurer l'isolement du circuit RRI lors d'une fuite de fluide primaire (notamment pour les pressions et températures correspondantes et pour des mélanges diphasiques eau-vapeur), une rupture du circuit RRI peut intervenir dans l'enceinte de confinement ou à l'extérieur de celle-ci dans des portions de circuit en dehors du tronçon compris entre le clapet antiretour et la vanne.

La fréquence estimée de fusion du cœur avec bipasse du confinement lors d'une telle rupture du circuit RRI dépend de la capacité de la vanne d'isolement RRI à se fermer. Compte tenu des grandes incertitudes sur cette capacité, l'ASN a, dans le cadre du réexamen de sûreté associé aux troisièmes visites décennales des tranches de $900 \mathrm{MWe}$, demandé la mise en œuvre d'une modification de conception permettant de garantir la disponibilité du circuit RRI en cas de rupture du serpentin de la barrière thermique.

Pour les réacteurs de $1450 \mathrm{MWe}$, à l'issue de leurs premières visites décennales, l'ASN a demandé à l'exploitant de présenter en 2013 une analyse approfondie des scénarios accidentels de ce type.

Pour les réacteurs de 1300 MWe, ce sujet est en cours d'examen dans le cadre du réexamen de sûreté associé aux troisièmes visites décennales.

Pour le réacteur EPR, la fréquence estimée de fusion du cœur est très faible du fait que l'isolement du circuit RRI est qualifié pour être réalisable dans le cas d'une rupture guillotine d'un serpentin et assuré par deux vannes diversifiées. De plus, par conception, en cas de défaillance de cet isolement, la perte du RRI ne peut être que partielle.

\subsubsection{Bipasses de l'enceinte de confinement pouvant survenir lors d'un accident de dimensionnement}

Sont présentés ici les bipasses de l'enceinte de confinement associés à des ruptures de tubes de générateurs de vapeur (RTGV).

La rupture d'un tube de générateur de vapeur (RTGV) conduit à l'arrêt automatique du réacteur et à la mise en service automatique de l'injection de sécurité et de l'alimentation de secours en eau des générateurs de vapeur (ASG). Les principales actions à réaliser par l'opérateur consistent à :

- identifier le générateur de vapeur affecté et l'isoler du côté secondaire. Cet isolement doit être effectué rapidement pour éviter le remplissage en eau du 
générateur de vapeur et donc la sollicitation en eau des vannes de décharge ou des soupapes de sûreté associées ;

- refroidir le réacteur par les générateurs de vapeur sains et abaisser la pression du circuit primaire de manière à annuler la fuite primaire-secondaire le plus rapidement possible puis à rallier les conditions de mise en service du système RRA. L'arrêt de l'injection de sécurité par les opérateurs, effectué sur des critères précis, est nécessaire pour limiter le remplissage en eau du générateur de vapeur et pour permettre la dépressurisation du circuit primaire.

La conduite vers l'état d'arrêt à froid pour intervention est poursuivie par la mise en service du circuit de refroidissement du réacteur à l'arrêt (RRA).

À la suite d'une rupture de tubes d'un générateur de vapeur, un bipasse du confinement peut résulter d'une fuite de fluide primaire par les vannes de décharge ou les soupapes de sûreté du circuit secondaire survenant après le remplissage du côté secondaire du générateur de vapeur affecté par le fluide primaire. Une telle situation peut se produire en cas d'isolement tardif du générateur de vapeur affecté ou d'arrêt tardif de l'injection de sécurité par l'opérateur et de l'absence de refermeture des vannes ou des soupapes du circuit secondaire après leur fonctionnement en eau, suivi de la défaillance du système RRA lors de sa mise en service ou de son fonctionnement, ce qui entraîne la vidange complète de la bâche PTR à l'extérieur de l'enceinte de confinement et donc l'impossibilité d'assurer une injection d'eau dans le circuit primaire.

Afin de réduire le risque de cumul d'une rupture de tubes d'un générateur de vapeur et d'une défaillance d'une soupape secondaire, des modifications ont été mises en place pour les réacteurs en exploitation en vue de limiter le remplissage du côté secondaire du générateur de vapeur affecté avec de l'eau du circuit primaire afin d'éviter le débordement en eau du générateur de vapeur. Notons que la situation est plus favorable pour les tranches de $1300 \mathrm{MWe}$ et de $1450 \mathrm{MWe}$ ainsi que pour le réacteur EPR, du fait que la pression de refoulement du circuit RIS (ISMP pour le réacteur EPR, voir le paragraphe 6.4.1) est moins élevée que pour les tranches de $900 \mathrm{MWe}$. Les résultats des EPS montrent que la probabilité de fusion du cœur avec bipasse du confinement associée à une rupture de tubes d'un générateur de vapeur est très faible (inférieure à $10^{-8}$ par année.réacteur pour les réacteurs de $900 \mathrm{MWe}$ ).

\subsubsection{Bipasses de l'enceinte de confinement pouvant survenir lors d'un accident de fusion du cœur}

\subsubsection{Ruptures induites de tubes de générateurs de vapeur}

Un des risques majeurs liés aux accidents de fusion du cœur se produisant à haute pression est celui d'un bipasse de l'enceinte de confinement à la suite de ruptures de tubes de générateurs de vapeur.

Les études existantes relatives à la fusion du cœur en pression, notamment celles réalisées à l'IRSN, montrent que la défaillance du circuit primaire interviendrait en premier lieu au niveau des tubes des générateurs de vapeur (c'est-à-dire avant une défaillance 
des branches chaudes ou une défaillance de la cuve) lorsque ceux-ci sont dépressurisés (§ 5.1.4). De plus, le retour d'expérience d'exploitation a montré que certains tubes des générateurs de vapeur sont fragilisés par le fonctionnement du réacteur (fragilisation par des mécanismes de corrosion et de fatigue). Malgré les dispositions prises pour contrôler l'état des tubes et isoler les tubes fragilisés ou corrodés (§ 2.4.2.3), l'existence de tubes fragilisés qui constituent des points faibles mécaniques de l'enveloppe du circuit primaire ne peut pas être exclue.

Des dispositions visant à éviter un accident de fusion du cœur en pression ont été prises compte tenu des conséquences possibles de ce type d'accident sur la troisième et dernière barrière de confinement, notamment en cas d'échauffement direct des gaz de l'enceinte ( $\mathrm{DCH}, \S 5.2 .1)$. Ces dispositions incluent une dépressurisation volontaire du circuit primaire par ouverture des vannes de décharge du pressuriseur. L'action de dépressurisation du circuit primaire est inscrite dans les procédures de conduite accidentelle et est à réaliser de manière immédiate par les opérateurs de conduite dès le début de l'utilisation du guide d'intervention en accident grave (GIAG, § 4.3.3.4).

Il est à noter qu'une modification de la commande d'ouverture des vannes de décharge du pressuriseur a été décidée pour les réacteurs de 900 MWe dans le cadre du réexamen de sûreté associé aux troisièmes visites décennales de façon à fiabiliser leur fonctionnement et donc la possibilité de dépressuriser le circuit primaire lors d'un accident avec fusion du cœur.

Pour le réacteur EPR, des dispositions de conception ont été prises visant à éliminer pratiquement les accidents de fusion du cœur en pression. Celles-ci ont été décrites au paragraphe 4.3.4.2.

Il convient de se reporter au paragraphe 5.1.4 pour plus de détails concernant la fusion du cœur en pression et les «brèches induites ».

\subsubsection{Rupture d'un circuit constituant une extension de la troisième barrière de confinement}

Les circuits appelés « extension de la troisième barrière de confinement » (E3B) sont des circuits nécessaires à la gestion d'une situation accidentelle rencontrée, dont la mise en service nécessite l'ouverture d'organes d'isolement de l'enceinte. Ces circuits peuvent conduire, en cas de défaillance (fuite ou rupture de tuyauterie, etc.), à des transferts de substances radioactives dans les bâtiments périphériques du bâtiment du réacteur ou dans l'environnement.

Pour les tranches de $900 \mathrm{MWe}$, les circuits extension de la troisième barrière de confinement lors d'un accident de fusion du cœur sont notamment :

- la portion du circuit d'éventage-filtration de l'enceinte de confinement comprise entre la vanne d'isolement de l'enceinte et la sortie du filtre à sable. Ce circuit et son utilisation sont décrits au paragraphe 4.3.3.3 ; 
- les portions des circuits RIS et EAS extérieures à l'enceinte, qui véhiculent de l'eau contaminée en provenance des puisards situés au fond du bâtiment réacteur lorsque ces circuits sont utilisés en recirculation (§ 2.4.2).

La mise en service d'un circuit E3B peut être décidée en application du guide d'intervention en accident grave (GIAG) ; elle a également pu être décidée plus tôt lors du déroulement de l'accident, dans le cadre de l'application du guide d'action des équipes de crise (GAEC).

La définition de l'extension de la troisième barrière de confinement, la liste des circuits extension de la troisième barrière de confinement, ainsi que les exigences à appliquer à ces circuits font l'objet d'un examen approfondi de l'IRSN et d'EDF notamment lors des réexamens de sûreté.

Il convient à cet égard de préciser que la notion de circuit extension de la troisième barrière de confinement est apparue après la conception des installations. De ce fait, pour les réacteurs en exploitation, la démarche de transposition des exigences associées à la troisième barrière de confinement à ses extensions a été menée dans une logique de vérification et non pas de conception. Cette démarche a visé en premier lieu à vérifier l'aptitude des circuits E3B, tels que conçus à :

- résister mécaniquement aux sollicitations induites par leur fonction ; il s'agit en fait d'une vérification par des études mécaniques réalisées sur l'ensemble des paliers ;

- assurer le confinement des substances radioactives pour toutes les situations pour lesquelles ils sont sollicités, notamment pour les situations accidentelles qui requièrent leur utilisation.

Cet examen par l'IRSN des exigences appliquées aux circuits E3B dans le cadre des réexamens de sûreté associés aux troisièmes visites décennales des réacteurs de $900 \mathrm{MWe}$ et aux deuxièmes visites décennales des réacteurs de $1300 \mathrm{MWe}$ a conduit à des demandes de vérification de tenue mécanique sur certains circuits pouvant véhiculer en situation accidentelle un mélange eau-vapeur (notamment sur les circuits REN/RCP) et de tenue à l'irradiation de certains équipements (notamment les joints nécessaires à l'étanchéité des circuits E3B).

Cet examen a également conduit à la mise en œuvre de modifications matérielles et de procédures visant d'une part à assurer une meilleure détection des défaillances possibles des circuits E3B, d'autre part à limiter les conséquences en cas de défaillance de ces circuits.

En ce qui concerne la surveillance de l'étanchéité des circuits E3B (notamment des circuits RIS et EAS), des chaînes de mesures d'activité (chaînes de mesure KRT) supplémentaires ainsi que des mesures de niveau d'eau dans les puisards du bâtiment des auxiliaires nucléaires, du bâtiment combustible et du bâtiment des auxiliaires de sauvegarde ont été intégrées dans les installations.

En ce qui concerne les moyens de limitation des conséquences en cas de défaillance de ces circuits, la stratégie actuelle d'EDF consiste, que ce soit pour les accidents de 
dimensionnement ou les accidents avec fusion du cœur, à réinjecter dans l'enceinte de confinement les effluents contaminés récupérés hors de l'enceinte. L'analyse des modifications associées à cette stratégie, déjà effectuée pour le palier 900 MWe dans le cadre des troisièmes visites décennales de ces réacteurs, est actuellement en cours à l'IRSN pour le palier 1300 MWe dans le cadre du réexamen de sûreté associé aux troisièmes visites décennales de ces réacteurs.

\subsection{Conclusion}

Comme l'a montré l'accident de TMI aux États-Unis (§ 7.1), l'enceinte de confinement constitue une barrière de confinement efficace lors d'un accident de fusion du cœur lorsque son intégrité et son étanchéité peuvent être assurées. Étudier les modes possibles de défaillance de l'enceinte de confinement et évaluer les moyens d'y faire face contribuent à réduire les possibilités de rejets radioactifs dans l'environnement pour toutes les situations d'accidents envisageables, y compris pour les accidents de fusion du cœur.

Comme nous l'avons vu dans ce chapitre, les études relatives au confinement consistent en un examen systématique des modes de défaillance possibles dans toutes les conditions de fonctionnement envisageables : défaillance de l'étanchéité de l'enceinte du bâtiment du réacteur elle-même, défaillance de l'étanchéité des traversées de cette enceinte, défaillance des circuits connectés au circuit primaire qui constituent dans certaines conditions de fonctionnement des « extensions de l'enceinte de confinement ».

L'objectif de ces études est en particulier d'améliorer les dispositions de conception et de conduite mises en œuvre sur les enceintes du parc en exploitation de façon à éviter autant que faire se peut la possibilité de rejets importants dans l'environnement en cas d'accident avec fusion du cœur. Pour les réacteurs actuellement en exploitation, les études ont ainsi notamment permis d'améliorer l'étanchéité du tampon d'accès des matériels en modifiant la boulonnerie associée.

À la conception du réacteur EPR, un objectif plus exigeant a été retenu, il s'agit en effet de " pratiquement éliminer » les accidents avec fusion du cœur qui conduiraient à des rejets précoces importants.

\section{Références}

[1] B. Masson, Tenue du confinement, évaluation des transferts à travers la paroi de l'enceinte, réunion sur la recherche et développement concernant les accidents graves, SFEN, Cadarache, France, 2009.

[2] E. Raimond, B. Laurent, R. Meignen, G. Nahas, B. Cirée, Advanced Modelling and Response Surface Method for Physical Models of level 2 PSA event tree, CSNIWG-RISK-Workshop level 2PSA and Severe Accident Management, Köln, Germany, 2004. 
[3] B. Cirée, G. Nahas, Mechanical analysis of the equipment hatch behaviour for the French PWR 900 MWe under severe accident, H01/3 - Proc. SMiRT, Toronto, Canada, 2007.

[4] G. Nahas, B. Cirée, Mechanical analysis of the containment building behaviour for the French PWR 900 MWe under severe accident, H05/5 - Proc. SMiRT, Toronto, Canada, 2007.

[5] M.F. Hessheimer, R.A. Dameron, Containment Integrity Research at Sandia National Laboratories, NUREG/CR-6906 SAND2006-2274P, 2006.

[6] International Standard Problem No 48, Containment capacity, Phase 2 Report Results of Pressure Loading Analysis, Organization for Economic Cooperation and Development, Nuclear Energy Agency, Committee on the Safety of Nuclear Installations, NEA/CSNI/R(2004)11, 2004.

[7] International Standard Problem N48, Containment capacity, Synthesis Report, Organization for Economic Cooperation and Development, Nuclear Energy Agency, Committee on the Safety of Nuclear Installations, NEA/CSNI/R(2005)5/ Vol. 1, 2 and 3, 2005. 